\title{
Thermal diffusivity and butterfly velocity in anisotropic Q-lattice models
}

\author{
Hyun-Sik Jeong, ${ }^{a}$ Yongjun Ahn, ${ }^{a}$ Dujin Ahn, ${ }^{a}$ Chao Niu, ${ }^{a}$ Wei-Jia Li ${ }^{b}$ \\ and Keun-Young $\mathrm{Kim}^{a}$ \\ ${ }^{a}$ School of Physics and Chemistry, Gwangju Institute of Science and Technology, \\ 123 Cheomdan-gwagiro, Gwangju 61005, Korea \\ ${ }^{b}$ Institute of Theoretical Physics, School of Physics, Dalian University of Technology, \\ No.2 Linggong Road, Dalian 116024, China \\ E-mail: hyunsik@gist.ac.kr, yongjunahn619@gmail.com, \\ dujinahn@gmail.com, chaoniu09@gmail.com, weijiali@dlut.edu.cn, \\ fortoe@gist.ac.kr
}

ABSTRACT: We study a relation between the thermal diffusivity $\left(D_{T}\right)$ and two quantum chaotic properties, Lyapunov time $\left(\tau_{L}\right)$ and butterfly velocity $\left(v_{B}\right)$ in strongly correlated systems by using a holographic method. Recently, it was shown that $\mathbb{E}_{i}:=D_{T, i} /\left(v_{B, i}^{2} \tau_{L}\right)$ $(i=x, y)$ is universal in the sense that it is determined only by some scaling exponents of the IR metric in the low temperature limit regardless of the matter fields and ultraviolet data. Inspired by this observation, by analyzing the anisotropic IR scaling geometry carefully, we find the concrete expressions for $\mathbb{E}_{i}$ in terms of the critical dynamical exponents $z_{i}$ in each direction, $\mathbb{E}_{i}=z_{i} / 2\left(z_{i}-1\right)$. Furthermore, we find the lower bound of $\mathbb{E}_{i}$ is always $1 / 2$, which is not affected by anisotropy, contrary to the $\eta / s$ case. However, there may be an upper bound determined by given fixed anisotropy.

KEYWORDS: Holography and condensed matter physics (AdS/CMT), Gauge-gravity correspondence

ARXIV EPRINT: 1708.08822 


\section{Contents}

1 Introduction 1

2 IR analysis for anisotropic Q-lattice models 4

2.1 General structure of the IR solutions 6

2.2 Marginally relevant axion 8

2.2.1 Class I: marginally relevant charge $\quad 8$

$\begin{array}{ll}2.2 .2 \text { Class II: irrelevant charge } & 10\end{array}$

$\begin{array}{lll}2.3 & \text { The other classes } & 13\end{array}$

$\begin{array}{ll}\text { 2.3.1 Irrelevant axion: class III and VI } & 13\end{array}$

$\begin{array}{lll}2.3 .2 & \text { Mixed axions } & 14\end{array}$

3 Thermal diffusion and butterfly velocity 14

$\begin{array}{lll}4 \text { Conclusion } & 21\end{array}$

A Irrelevant axion $\quad \mathbf{2 3}$

A.1 Class III: marginally relevant charge 23

A.2 Class IV: irrelevant charge 24

B Marginally relevant and irrelevant axion $\quad 25$

B.1 Class I-i: marginally relevant charge 25

B.2 Class I-ii: marginally relevant charge 26

B.3 Class II-i: irrelevant charge 28

$\begin{array}{ll}\text { B.4 Class II-ii: irrelevant charge } & 29\end{array}$

$\begin{array}{ll}\text { C Consistency check by coordinate transformation } & 31\end{array}$

\section{Introduction}

Strongly correlated electron systems are characterized by exotic ('strange') properties in contrast to weakly interacting systems. Interestingly enough, some exotic properties show a remarkable degree of universality [1]. For example, resistivity $(\rho)$ is observed to be linear in temperature $(T), \rho \sim T$, universally in various strange metals such as cuprates, pnictides and heavy fermions. It is in contrast to $\rho \sim T^{2}$ which can be explained by the Fermi liquid theory for metals with weakly interacting electrons. The strange metal state may undergo a phase transition to the high temperature superconducting state, where another universal property, Home's law [2-4], has been observed. It is a relation between three quantities: the superfluid density at zero temperature $\rho_{s}(T=0)$, the critical temperature $\left(T_{c}\right)$, and the DC electric conductivity right above the critical temperature $\left(\sigma_{\mathrm{DC}}\left(T_{c}\right)\right)$. The Homes' 
law states that $\rho_{s}(T=0) /\left(\sigma_{\mathrm{DC}}\left(T_{c}\right) T_{c}\right)$ is universal, which means it is independent of the components and structures of superconducting materials.

While such interesting properties in strongly correlated systems are difficult to analyze theoretically, the holographic methods (or the gauge/gravity duality) $[1,5,6]$ have provided novel and effective tools to investigate them. It maps strongly correlated systems to corresponding classical gravitational systems in higher dimensional spacetime, so 'holographic'. For example, for linear- $T$-resistivity, a lot of achievements including methodologies are reviewed in [1]. For the Homes' law, see [4, 7-9]. In general, universality in holography is related to the universal nature of the black hole horizon, which may shed light on universality in strongly correlated systems. Conversely, investigating as many universal properties in strongly correlated systems as possible may be helpful in understanding the black hole physics better. This will again back-react to our understanding of strongly correlated systems.

In this paper, we investigate another universal property regarding the thermal diffusivity and two quantum chaotic properties, butterfly velocity and Lyapunov time, from the holographic perspective. The thermal diffusivity $\left(D_{T}\right)$ is defined by

$$
D_{T}:=\frac{\kappa}{c_{\rho}},
$$

where $c_{\rho}$ is the specific heat at finite density and $\kappa$ is the thermal conductivity in the open circuit condition, i.e. at zero electric current. It was proposed that the thermal diffusivity has an interesting connection to the quantum chaos property as follows: ${ }^{1}$

$$
D_{T}=\mathbb{E} v_{B}^{2} \tau_{L}
$$

where $v_{B}$ is the butterfly velocity which describes the speed at which chaos propagates in space $[11,12,17-25]$, and $\tau_{L}$ is the Lyapunov time which measures the rate at which chaos grows in time. $\mathbb{E}$ is constant of order one. It was shown $[20]$ that there is a universal lower bound for $\tau_{L}$ :

$$
\tau_{L} \geq \frac{1}{2 \pi} \frac{\hbar}{k_{B} T}=: \frac{1}{2 \pi} \tau_{P},
$$

where the timescale $\left(\tau_{P}\right)$ was introduced in $[3,26]$ as the 'Planckian' dissipation time scale, which is the shortest possible time scale for dissipation. This time scale was observed in the scattering rates of materials having a linear $T$ resistivity [27] and in the thermal diffusivity [28]. The Lyapunov time saturates the bound in holographic theories with Einstein gravity. While the connection (1.2) between transport properties and chaos was first proposed in the holographic models, it has been also observed in condensed matter theories [28-31].

The relation (1.2) is shown to be universal in several cases. It was shown that, in a class of holographic model with a scaling infra-red (IR) geometry characterized by critical exponents such as dynamical critical exponent $(z)$, hyperscaling violating exponent $(\theta)$ or charge

\footnotetext{
${ }^{1}$ This kind of relation was first motivated by the charge diffusivity and its relation to the linear- $T$ resistivity [10-12]. However, it turned out the relation (1.2) for charge diffusivity does not hold in many models, for example, striped holographic matter [13], the SYK model [14], higher derivative models [15] and the Gubser-Rocha model [16].
} 
anomalous parameter $(\zeta)[32], \mathbb{E}$ is a function only of a dynamical critical exponent $z \neq 1$

$$
\mathbb{E}=\frac{1}{2} \frac{z}{z-1},
$$

at zero density in the low temperature limit, independently of other critical exponents, momentum relaxation strength and UV data [12]. ${ }^{2}$ Recently this analysis was extended to finite density or magnetic field case and $\mathbb{E}$ is shown to be independent of charge density and magnetic field too [34]. More evidences for (1.2) have been reported in holographic models that flow to $\mathrm{AdS}_{2} \times R^{d}$ fixed points in the IR [33] and in the higher derivative model $[15,35,36]$. At finite density, the thermal diffusivity is not an eigenvalue of the diffusivity matrix because of its mixing with the charge diffusivity. However, it was shown in [16] that the mixing effect becomes negligible in the incoherent regime (i.e. the regime of strong momentum relaxation) and $\mathbb{E}$ becomes universal in that regime even at finite density. More interestingly, while the relation (1.2) was first proposed in the holographic models, it has been also observed in condensed matter theories [28-31, 37, 38] including the Sachdev-Ye-Kitaev (SYK) models [14, 39, 40].

In this paper, we want to study the effect of spatial anisotropy ${ }^{3}$ on the universality of $\mathbb{E}$, more precisely, two $\mathbb{E}$ 's, one for the $x$-direction $\left(\mathbb{E}_{x}\right)$ and one for the $y$-direction $\left(\mathbb{E}_{y}\right)$. This question was already addressed in [34], where it was shown that both $\mathbb{E}_{x}$ and $\mathbb{E}_{y}$ are determined only by some scaling parameters $\left(u_{1}, v_{1}, v_{2}\right)$ near horizon:

$$
\mathbb{E}_{x}=\frac{u_{1}-1}{u_{1}-2 v_{1}}, \quad \mathbb{E}_{y}=\frac{u_{1}-1}{u_{1}-2 v_{2}} .
$$

Our goal here is to extend these results in three aspects. i) to understand $\mathbb{E}_{x}$ and $\mathbb{E}_{y}$ in terms of scaling exponents $z$ and the anisotropic parameter $\xi$ and ii) to identify the allowed range of $\mathbb{E}_{x}$ and $\mathbb{E}_{y}$ and see if there is any universal lower or upper bound. iii) to extend the formalism in [34] to the case where $g_{t t} \neq g_{r r}^{-1}$. To achieve our goals, we first have performed the complete analysis of the IR geometry of the anisotropic Q-lattice models. Note that this study itself is a useful investigation on its own apart from the application to diffusion and butterfly velocity because the Q-lattice models are widely used in various aspects of AdS/CMT applications. In this respect, our work amounts to the anisotropic extension of the isotropic Q-lattice models in [32].

We find the concrete expression for $\mathbb{E}_{i}$ in terms of the dynamical exponents $z_{i}$ for $i$-direction $(i=x, y)$ :

$$
\mathbb{E}_{x}=\frac{1}{2} \frac{z_{x}}{z_{x}-1}, \quad \mathbb{E}_{y}=\frac{1}{2} \frac{z_{y}}{z_{y}-1},
$$

which clearly show universality in terms of physical parameters and the effect of anisotropy. $\mathbb{E}_{x}$ and $\mathbb{E}_{y}$ do not depend on other critical exponents $(\theta, \zeta)$ and charge density $\rho$. This

\footnotetext{
${ }^{2}$ In this work, it is assumed that either the charge density or the axion fields are marginally relevant (of which meaning will be explained below (2.23)), which amounts to $z \neq 1$. It is also assumed that the dilaton field $\varphi(r)$ behaves as $\log r$ at IR so does not approach to a constant value, which means that the IR geometry is not $\mathrm{AdS}_{2} \times R^{p-1}$. For more discussions for the IR geometry of $z=1$ and/or $\operatorname{AdS}_{2} \times R^{p-1}$ we refer to $[33,34]$.

${ }^{3}$ For the effect of spatial anisotropy on shear viscosity, see [41].
} 
universality is due to nontrivial cancellations between three quantities $\left\{\kappa, c_{\rho}, v_{B}\right\}$, all of which depend on many other parameters including UV data.

So far we discussed the universality of $\mathbb{E}_{i}$ in the sense that $\mathbb{E}_{i}$ is independent of many IR parameters as well as UV data. However, it is also interesting to see if there is any universal lower or upper ${ }^{4}$ bound of $\mathbb{E}_{i}$. For example, in the case of (1.4) and (1.6) it amounts to asking if there is any universal bound of dynamical critical exponents $z_{i}$. For the isotropic IR scaling geometry it was shown that $z>1$ [32], which implies ${ }^{5}$

$$
\frac{1}{2} \leq \mathbb{E}
$$

where $\mathbb{E}$ saturates its minimum value $\mathbb{E}=1 / 2$ as $z \rightarrow \infty$. For the theories that flow to $\mathrm{AdS}_{2} \times R^{d}$ IR fixed points, $\mathbb{E}$ depends only on the leading irrelevant mode and $1 / 2<\mathbb{E} \leq 1$ where $\mathbb{E}=1$ if the leading deformation is a dilatonic mode [33]. It matches the value in extended SYK models $[14,39]$. However, it was reported that $\mathbb{E}$ may not have a universal lower bound in an inhomogeneous SYK model [42] or in a higher derivative gravity theory [45].

Here we investigate if the range (1.7) for the isotropic scaling geometry can be affected by anisotropy. It was motivated by a series of works regarding the universal bound of sheer viscosity to entropy density ratio $(\eta / s)$ so called the KSS(Kovtun, Starinets and Son) bound, $1 / 4 \pi$ [46]. It can be lowered further by the higher derivative gravity [47, 48]. However, it can even vanish by anisotropy at zero temperature ${ }^{6}$ [53-56]. In our anisotropic model, we find that the lower bound of $\mathbb{E}_{i}$ is always $1 / 2$ regardless of anisotropy but the upper bound of $\mathbb{E}_{i}$ may depend on anisotropy. In other words, $z_{i}$ depends on anisotropy.

This paper is organized as follows. In section 2, we introduce the 'Q-lattice model' or the Einstein-Maxwell-Dilaton theory coupled to 'axion' fields. By assuming specific couplings in the IR region, we obtain the IR scaling solutions described by four scaling parameters, and classify the solutions according to the IR relevance of gauge field and axion field. We also analyze the allowed parameter region of the solutions by requiring some physical conditions. In section 3 we study the thermal diffusivity, butterfly velocity, and their universality based on the results obtained in section 2 . In section 4 , we conclude.

\section{IR analysis for anisotropic Q-lattice models}

Let us consider the 'Q-lattice action'

$$
\begin{aligned}
S & =\int \mathrm{d}^{p+1} x \sqrt{-g}\left(R+\mathcal{L}_{m}\right), \\
\mathcal{L}_{m} & \equiv-\frac{1}{2}(\partial \varphi)^{2}+V(\varphi)-\frac{1}{4} Z(\varphi) F^{2}-\frac{1}{2} W_{1}(\varphi) \sum_{i=1}^{p-2}\left(\partial \chi_{i}\right)^{2}-\frac{1}{2} W_{2}(\varphi)\left(\partial \chi_{p-1}\right)^{2},
\end{aligned}
$$

which is the Einstein-Maxwell-Dilaton theory coupled to 'Axion' fields $\chi_{i}(i=1, \ldots, p-1)$. This model is also called the EMD-Axion action. We introduce the axions as many as

\footnotetext{
${ }^{4}$ The existence of the upper bound was proposed in $[42,43]$.

${ }^{5}$ Mathematically $z<0$ is allowed, but we do not consider it here: it is not physical because $\omega \sim k^{z}$ [44].

${ }^{6}$ Momentum relaxation gives similar results at zero temperature [49-52].
} 
spatial dimensions and every axion may have different coupling in general, say $W_{i}(\varphi)$. However, for simplicity we introduce anisotropy minimally by two couplings $W_{1}$ and $W_{2}$. We will further assume the axion fields have the form

$$
\chi_{i}=k_{1} x_{i} \quad(i=1, \ldots, p-2), \quad \chi_{p-1}=k_{2} y,
$$

to break translational symmetry. Here we introduced another anisotropy by $k_{1}$ and $k_{2}$. In summary, we introduced two kinds of anisotropy: i) in the action, $W_{1}$ and $W_{2}$ ii) in the solution $k_{1}$ and $k_{2}$.

The action yields the following Einstein equations:

$$
\begin{aligned}
R_{\mu \nu}= & T_{\mu \nu}-\frac{1}{p-1} g_{\mu \nu} T \\
= & \frac{1}{2} \partial_{\mu} \varphi \partial_{\nu} \varphi+\frac{W_{1}(\varphi)}{2} \sum_{i=1}^{p-2} \partial_{\mu} \chi_{i} \partial_{\nu} \chi_{i}+\frac{W_{2}(\varphi)}{2} \partial_{\mu} \chi_{p-1} \partial_{\nu} \chi_{p-1}+\frac{Z(\varphi)}{2} F_{\mu}{ }^{\rho} F_{\nu \rho} \\
& -\frac{Z(\varphi) F^{2}}{4(p-1)} g_{\mu \nu}-\frac{V(\varphi)}{p-1} g_{\mu \nu},
\end{aligned}
$$

where $T_{\mu \nu}=-\frac{1}{\sqrt{-g}} \frac{\delta\left(\sqrt{-g} \mathcal{L}_{m}\right)}{\delta g^{\mu \nu}}$ and $T=g^{\mu \nu} T_{\mu \nu}$. The Maxwell equation, scalar equation, and axion equation read

$$
\begin{aligned}
& \nabla_{\mu}\left(Z(\varphi) F^{\mu \nu}\right)=0, \\
& \square \varphi+V^{\prime}(\varphi)-\frac{1}{4} Z^{\prime}(\varphi) F^{2}-\frac{1}{2} W_{1}^{\prime}(\varphi) \sum_{i=1}^{p-2}\left(\partial \chi_{i}\right)^{2}-\frac{1}{2} W_{2}^{\prime}(\varphi)\left(\partial \chi_{p-1}\right)^{2}=0, \\
& \nabla_{\mu}\left(W_{1}(\varphi) \nabla^{\mu} \chi_{i}\right)=0, \quad \nabla_{\mu}\left(W_{2}(\varphi) \nabla^{\mu} \chi_{p-1}\right)=0 .
\end{aligned}
$$

By considering the following homogeneous (all functions are only functions of $r$ ) ansatz

$$
\begin{aligned}
\mathrm{d} s^{2} & =-D(r) \mathrm{d} t^{2}+B(r) \mathrm{d} r^{2}+C_{1}(r) \sum_{i=1}^{p-2} \mathrm{~d} x_{i}^{2}+C_{2}(r) \mathrm{d} y^{2}, \\
\varphi & =\varphi(r), \quad A=A_{t}(r) \mathrm{d} t, \quad \chi_{i}=k_{1} x_{i}, \quad \chi_{p-1}=k_{2} y,
\end{aligned}
$$

we obtain the Einstein equations

$$
\begin{aligned}
0= & \frac{Z(p-2) A_{t}^{\prime 2}}{(p-1) D}+\frac{2 B V}{p-1}+\frac{B^{\prime} D^{\prime}}{2 B D}-\frac{(p-2) D^{\prime} C_{1}^{\prime}}{2 D C_{1}}-\frac{D^{\prime} C_{2}^{\prime}}{2 D C_{2}}+\frac{D^{\prime 2}}{2 D^{2}}-\frac{D^{\prime \prime}}{D}, \\
0= & \varphi^{\prime 2}-\frac{(p-2) C_{1}^{\prime 2}}{2 C_{1}^{2}}-\frac{C_{2}^{\prime 2}}{2 C_{2}^{2}}-\frac{D^{\prime}}{2 D}\left(\frac{(p-2) C_{1}^{\prime}}{C_{1}}+\frac{C_{2}^{\prime}}{C_{2}}\right)-\frac{B^{\prime}}{2 B}\left(\frac{(p-2) C_{1}^{\prime}}{C_{1}}+\frac{C_{2}^{\prime}}{C_{2}}\right) \\
& +\frac{(p-2) C_{1}^{\prime \prime}}{C_{1}}+\frac{C_{2}^{\prime \prime}}{C_{2}}, \\
0= & \frac{W_{1} k_{1}^{2} B}{C_{1}}-\frac{2 B V}{p-1}+\frac{Z A_{t}^{\prime 2}}{(p-1) D}+\frac{C_{1}^{\prime}}{2 C_{1}}\left(\frac{D^{\prime}}{D}-\frac{B^{\prime}}{B}\right)+\frac{(p-4) C_{1}^{\prime 2}}{2 C_{1}^{2}}+\frac{C_{1}^{\prime} C_{2}^{\prime}}{2 C_{1} C_{2}}+\frac{C_{1}^{\prime \prime}}{C_{1}}, \\
0= & \frac{W_{2} k_{2}^{2} B}{C_{2}}-\frac{2 B V}{p-1}+\frac{Z A_{t}^{\prime 2}}{(p-1) D}+\frac{C_{2}^{\prime}}{2 C_{2}}\left(\frac{D^{\prime}}{D}-\frac{B^{\prime}}{B}\right)-\frac{C_{2}^{\prime 2}}{2 C_{2}^{2}}+\frac{(p-2) C_{1}^{\prime} C_{2}^{\prime}}{2 C_{1} C_{2}}+\frac{C_{2}^{\prime \prime}}{C_{2}},
\end{aligned}
$$


which come from the equations corresponding to $R_{t t}, R_{r r}, R_{x x}$, and $R_{y y}$ in (2.3) respectively. The prime' denotes the derivative with respect to $r$. The Maxwell equation and scalar equation are reduced to

$$
\begin{aligned}
0= & {\left[Z \frac{C_{1}^{\frac{p-2}{2}} C_{2}^{\frac{1}{2}}}{\sqrt{B D}} A_{t}^{\prime}\right]^{\prime}, } \\
0= & -\frac{W_{1, \varphi} k_{1}^{2}(p-2) B}{2 C_{1}}-\frac{W_{2, \varphi} k_{2}^{2} B}{2 C_{2}}+\frac{Z_{, \varphi} A_{t}^{\prime 2}}{2 D}+B V_{, \varphi}-\frac{B^{\prime} \varphi^{\prime}}{2 B} \\
& +\left(\frac{(p-2) C_{1}^{\prime}}{2 C_{1}}+\frac{C_{2}^{\prime}}{2 C_{2}}\right) \varphi^{\prime}+\frac{D^{\prime} \varphi^{\prime}}{2 D}+\varphi^{\prime \prime},
\end{aligned}
$$

and the axion equations are satisfied trivially.

\subsection{General structure of the IR solutions}

In this paper, we are mainly interested in the scaling geometry at IR, where $\varphi$ runs logarithmically and the dilaton couplings are approximated as

$$
Z(\varphi) \sim e^{\gamma \varphi}, \quad V(\varphi) \sim V_{0} e^{-\delta \varphi}, \quad W_{1}(\varphi) \sim e^{\lambda_{1} \varphi}, \quad W_{2}(\varphi) \sim e^{\lambda_{2} \varphi} .
$$

Here we introduce parameters $\left(\gamma, \delta, \lambda_{1}, \lambda_{2}, V_{0}\right)$, which we call 'action-parameters'. For $Z, W_{1}, W_{2}$ we do not introduce the coefficients because they can be absorbed into the gauge field and axions. To analyze the IR solution, we will plug the IR couplings (2.12) into (2.6)-(2.11) and assume that the IR solutions are written as

$$
\begin{aligned}
\mathrm{d} s^{2} & =r^{\frac{2 \theta}{p-1}}\left[-\frac{\mathrm{d} t^{2}}{r^{2 z}}+\frac{L_{r}^{2} \mathrm{~d} r^{2}}{r^{2}}+\frac{L_{1}^{2} \sum_{i=1}^{p-2} \mathrm{~d} x_{i}^{2}}{r^{2 \xi_{x}}}+\frac{L_{2}^{2} \mathrm{~d} y^{2}}{r^{2 \xi_{y}}}\right], \\
\varphi & =\varphi_{0} \log r, \quad A=a_{0} r^{\zeta-z} \mathrm{~d} t, \quad \chi_{i}=k_{1} x_{i}, \quad \chi_{p-1}=k_{2} y,
\end{aligned}
$$

in terms of 'exponents' $\left(z, \theta, \xi_{x}, \xi_{y}, \zeta\right)$ and 'coefficients' $\left(\varphi_{0}, a_{0}, L_{r}, L_{1}, L_{2}, k_{1}, k_{2}\right)$. We will call all of them 'solution-parameters', to explain their relations to the 'action-parameters' which are the parameters in the action. For example, by the equations of motion, the exponent-solution-parameters $\left(z, \theta, \xi_{x}, \xi_{y}, \zeta\right)$ will be related to the action parameters $(\gamma, \delta$, $\left.\lambda_{1}, \lambda_{2}\right)$. Notice that this kind of scaling solutions (2.13) are possible since the scalar is of the form $\left(e^{\varphi}=r^{\varphi_{0}}\right)$.

Some of solution-parameters are redundant and can be set to unity by coordinate transformations. Depending on our purpose and perspective we may choose independent parameters without loss of generality. In this paper, we will choose the representation with

$$
\xi_{x}=1, \quad \xi_{y}=\xi,
$$

for an easy comparison with the isotropic results obtained in [32]. ${ }^{7}$ We will mostly fix $L_{1}=L_{2}=1$ but sometimes we find it more convenient to keep $L_{1}$ and $L_{2}$ unfixed.

\footnotetext{
${ }^{7}$ We may have chosen the convention $\xi_{x}=\xi_{y}=\xi$ for the isotropic case. However, without loss of generality we can choose (2.14), where the isotropic case corresponds to $\xi=1$. With this choice, we can easily compare all of our formulas with the isotropic cases in [32].
} 
The metric is parameterized in a way to identify four critical exponents. There are two dynamical critical exponents

$$
z_{x}:=\frac{z}{\xi_{x}}=z, \quad z_{y}:=\frac{z}{\xi_{y}}=\frac{z}{\xi} .
$$

They describe the anisotropy between time and space $x_{i}$, and time and space $y$ respectively, where $\xi$ quantifies the anisotropy between $x_{i}$-space and $y$-space. A hyperscaling violating exponent $\theta$ measures how much the scale invariance of the metric is violated and has something to do with the anomalous dimension of the field theory energy density. $\zeta$ describing the anomalous scaling of the bulk Maxwell field is related to the anomalous dimension of the field theory charge density.

Furthermore, it turns out that the emblackening factor $f(r)$

$$
f(r)=1-\left(\frac{r}{r_{h}}\right)^{z+p-2-\theta+\xi}
$$

can be turned on $\left(\mathrm{d} t^{2} \rightarrow f \mathrm{~d} t^{2}\right.$ and $\mathrm{d} r^{2} \rightarrow \mathrm{d} r^{2} / f$ in (2.13)) in all cases we consider in this paper.

Some of the solutions parameters are fixed by the action parameters by the equations of motion but some of them are not fixed and remain free. However, the range of all parameters should be restricted by the following conditions. First, for the IR geometry to be well-defined, we require

$$
\theta>(p-1) z, \quad \theta>p-1, \quad \theta>(p-1) \xi, \quad \theta>z+p-2+\xi,
$$

if the IR is located at $r \rightarrow 0$ or

$$
\theta<(p-1) z, \quad \theta<p-1, \quad \theta<(p-1) \xi, \quad \theta<z+p-2+\xi,
$$

if the IR is located at $r \rightarrow \infty$. The first three inequalities of (2.17) and (2.18) come from the condition that all metric components should vanish at the IR at zero $T$. The last inequalities come from the condition that the emblackening factor (2.16) should vanish at the UV. We also require ${ }^{8}$

$$
L_{r}^{2}>0, \quad L_{1}^{2}>0, \quad L_{2}^{2}>0,
$$

and the specific heat should be positive:

$$
\frac{-2 \theta+(p-1)(\xi+1)}{(p-1) z}>0
$$

which can be read from the scaling of entropy, $S \sim T^{\frac{-2 \theta+(p-1)(\xi+1)}{(p-1) z}}$. If all of the above conditions are satisfied we have confirmed that the following null energy condition (NEC) is also satisfied:

$$
\begin{array}{r}
((p-1)-\theta)((p-1)(z-1)-\theta)-(\xi-1)(2(\xi-1)+(2-z)(p-1)) \geq 0 \\
(z-1)(-\theta+\xi+p+z-2) \geq 0 \\
(z-1)(-\xi+z) \geq 0 .
\end{array}
$$

\footnotetext{
${ }^{8}$ If we choose the representation $L_{1}=L_{2}=1$, we need to consider other reality conditions equivalent to them.
} 
We categorize the solutions according to the 'relevance' of the axion and/or charge, following [32] for the easy comparison with the isotropic case therein. ${ }^{9}$ By 'marginally relevant axion' we mean the axion parameter $k_{1}, k_{2}$ appear explicitly in the leading solutions and by 'marginally relevant charge' we mean $a_{0}$ appears explicitly in the leading solutions. By 'irrelevant axion (charge)' we mean $k_{1}, k_{2}\left(a_{0}\right)$ do not appear explicitly in the leading solutions but they can appear in the sub-leading solutions. Therefore, we will consider eight classes as follows.

- class I: marginally relevant axions \& charge

$$
\begin{aligned}
& \left(k_{1} \neq 0, k_{2} \neq 0, a_{0} \neq 0\right) \\
& \left(k_{1} \neq 0, k_{2} \neq 0, a_{0}=0\right) \\
& \left(k_{1}=0, k_{2}=0, a_{0} \neq 0\right) \\
& \left(k_{1}=0, k_{2}=0, a_{0}=0\right) \\
& \left(k_{1} \neq 0, k_{2}=0, a_{0} \neq 0\right) \\
& \left(k_{1}=0, k_{2} \neq 0, a_{0} \neq 0\right) \\
& \left(k_{1} \neq 0, k_{2}=0, a_{0}=0\right) \\
& \left(k_{1}=0, k_{2} \neq 0, a_{0}=0\right)
\end{aligned}
$$$$
\text { - class II: marginally relevant axions \& irrelevant charge }
$$$$
\text { - class III: irrelevant axions \& marginally relevant charge }
$$$$
\text { - class IV: irrelevant axions \& charge }
$$$$
\text { - class I-i: mixed axions \& marginally relevant charge }
$$$$
\text { - class I-ii: mixed axions \& marginally relevant charge }
$$

- class II-i: mixed axions \& irrelevant charge

- class II-ii: mixed axions \& irrelevant charge

Here, 'mixed axions' means the axion in one direction is marginally relevant and the axion in the other direction is irrelevant. They reduce to four classes in [32] in the isotropic limit.

Notice that the classification is based on the property of the leading solutions. We also should consider the deformation by the sub-leading solutions:

$$
\Phi_{i} \rightarrow \Phi_{i}+\epsilon_{i} r^{\beta_{i}}+\cdots,
$$

where $\Phi_{i}$ denotes every leading order solution collectively and $\epsilon_{i}$ is a small parameter. Therefore, $a_{0}=0$ does not mean zero density and $k_{i}=0$ does not mean no momentum relaxation in the $i$-direction because these parameters can appear in the sub-leading solutions. If the axion is relevant, we may expect the momentum relaxation affects IR physics more strongly than the irrelevant axion cases.

\subsection{Marginally relevant axion}

\subsubsection{Class I: marginally relevant charge}

We assume that the classical solutions are written as

$$
\begin{aligned}
\mathrm{d} s^{2} & =r^{\frac{2 \theta}{p-1}}\left[-\frac{\mathrm{d} t^{2}}{r^{2 z}}+\frac{L_{r}^{2} \mathrm{~d} r^{2}}{r^{2}}+\frac{L_{1}^{2} \sum_{i=1}^{p-2} \mathrm{~d} x_{i}^{2}}{r^{2}}+\frac{L_{2}^{2} \mathrm{~d} y^{2}}{r^{2 \xi}}\right], \\
\varphi & =\varphi_{0} \log r, \quad A=a_{0} r^{\zeta-z} \mathrm{~d} t, \quad \chi_{i}=k_{1} x_{i}, \quad \chi_{p-1}=k_{2} y,
\end{aligned}
$$

\footnotetext{
${ }^{9}$ Compared to [32], we change the name from 'current' to 'charge' to be more specific to the charge density $J^{t}$ among the current operator $J^{\mu}$.
} 
where $\varphi_{0}, a_{0}, k_{1}$, and $k_{2}$ are nonzero and $\zeta \neq z$. Or, we may choose the following equivalent ansatz

$$
\begin{aligned}
\mathrm{d} s^{2} & =r^{\frac{2 \theta}{p-1}}\left[-\frac{\mathrm{d} t^{2}}{r^{2 z}}+\frac{L_{r}^{2} \mathrm{~d} r^{2}}{r^{2}}+\frac{\sum_{i=1}^{p-2} \mathrm{~d} \tilde{x}_{i}^{2}}{r^{2}}+\frac{\mathrm{d} \tilde{y}^{2}}{r^{2 \xi}}\right], \\
\varphi & =\varphi_{0} \log r, \quad A=a_{0} r^{\zeta-z} \mathrm{~d} t, \quad \chi_{i}=\tilde{k}_{1} \tilde{x}_{i}, \quad \chi_{p-1}=\tilde{k}_{2} \tilde{y}
\end{aligned}
$$

where

$$
\tilde{x}_{i} \equiv L_{1} x_{i}, \quad \tilde{y} \equiv L_{2} y, \quad \tilde{k}_{1} \equiv \frac{k_{1}}{L_{1}}, \quad \tilde{k}_{2} \equiv \frac{k_{2}}{L_{2}} .
$$

By the equations of motion, the 'exponent' solution-parameters $(z, \xi, \theta, \zeta)$ may be expressed in terms of action-parameters $\left(\delta, \lambda_{1}, \lambda_{2}, \gamma\right)$ as

$$
\begin{aligned}
& z=\frac{2-(p-1) \delta^{2}+(p-2) \lambda_{1}^{2}+\lambda_{2}^{2}}{\lambda_{1}\left((p-1) \delta+(p-2) \lambda_{1}+\lambda_{2}\right)} \\
& \theta=-\frac{(p-1) \delta}{\lambda_{1}}, \quad \xi=\frac{\lambda_{2}}{\lambda_{1}}, \quad \zeta=\frac{\gamma-\delta}{\lambda_{1}} .
\end{aligned}
$$

They are not all independent and there is a constraint between solution-parameters $(\theta, \zeta, \xi)$

$$
\zeta=-(p-2-\theta)-\xi \equiv-\left(d_{\theta}-1\right)-\xi \equiv \zeta_{I},
$$

which amounts to a relation between action-parameters:

$$
\gamma=(2-p) \delta+(2-p) \lambda_{1}-\lambda_{2} .
$$

The four 'coefficient' parameters are solved as

$$
\varphi_{0}=-\frac{2}{\lambda_{1}}
$$

and

$$
\begin{aligned}
a_{0}^{2}= & \frac{2\left(\tilde{k}_{1}^{2}(p-1)\left(\delta \lambda_{2}+\lambda_{2}^{2}+\lambda_{1}(p-2)\left(\delta+\lambda_{1}\right)+2\right)+2 V_{0}\left(-\lambda_{2}^{2}+\lambda_{1} \lambda_{2}+\delta(p-1)\left(\delta+\lambda_{1}\right)-2\right)\right)}{\left(\tilde{k}_{1}^{2}(p-2)-2 V_{0}\right)\left(2 \lambda_{2}^{2}+(p-2)(p-1)\left(\delta+\lambda_{1}\right)^{2}+2 \lambda_{2}\left(\delta(p-1)+\lambda_{1}(p-2)\right)+2\right)}, \\
L_{r}^{2}= & -\frac{2\left(\lambda_{2}^{2}+(p-2)(p-1)\left(\delta+\lambda_{1}\right)^{2}+\lambda_{2}\left(\delta(p-1)+\lambda_{1}(p-2)\right)+2\right)}{\lambda_{1}^{2}\left(\tilde{k}_{1}^{2}(p-2)-2 V_{0}\right)\left(\lambda_{2}+\delta(p-1)+\lambda_{1}(p-2)\right)^{2}} \\
& \times\left(2 \lambda_{2}^{2}+(p-2)(p-1)\left(\delta+\lambda_{1}\right)^{2}+2 \lambda_{2}\left(\delta(p-1)+\lambda_{1}(p-2)\right)+2\right), \\
\tilde{k}_{2}^{2}= & \tilde{k}_{1}^{2}-\frac{\left(\lambda_{1}-\lambda_{2}\right)\left(\tilde{k}_{1}^{2}(p-2)-2 V_{0}\right)\left(\lambda_{2}+\delta(p-1)+\lambda_{1}(p-2)\right)}{\lambda_{2}^{2}+(p-2)(p-1)\left(\delta+\lambda_{1}\right)^{2}+\lambda_{2}\left(\delta(p-1)+\lambda_{1}(p-2)\right)+2} .
\end{aligned}
$$

The action-parameters $\left(\delta, \lambda_{1}, \lambda_{2}, \gamma\right)$ may be written in terms of solution-parameters $(z, \theta, \xi)$ :

$$
\delta=\frac{2 \theta}{(p-1) \varphi_{0}}, \quad \lambda_{1}=\frac{-2}{\varphi_{0}}, \quad \lambda_{2}=\frac{-2 \xi}{\varphi_{0}}, \quad \gamma=\frac{-2 \zeta+\frac{2}{p-1} \theta}{\varphi_{0}}
$$


where

$$
\begin{aligned}
\varphi_{0}^{2} & =2\left(\frac{\theta^{2}}{p-1}-z \zeta+2-p-\xi^{2}\right) \\
& =\frac{2(p-1-\theta)(1+p(z-1)-z-\theta)}{p-1}-2(\xi-1)(\xi-z+1),
\end{aligned}
$$

where $\zeta$ can be replaced by (2.29). Using the relations (2.33), the equations (2.32) can be simplified as

$$
\begin{aligned}
L_{r}^{2} & =\frac{2(p-2+z-\theta)(p-2+z-\theta+\xi)}{2 V_{0}-(p-2) \tilde{k}_{1}^{2}}, \\
a_{0}^{2} & =\frac{2\left(2 V_{0}(1-z)+(z(p-1)-\theta) \tilde{k}_{1}^{2}\right)}{(2-p-z+\theta-\xi)\left(2 V_{0}-(p-2) \tilde{k}_{1}^{2}\right)} \\
\tilde{k}_{2}^{2} & =\tilde{k}_{1}^{2}+(\xi-1) \frac{\tilde{k}_{1}^{2}(p-2)-2 V_{0}}{p-2+z-\theta}
\end{aligned}
$$

where we choose $\tilde{k}_{1}$ as a free parameter. Note that the last equation can be written as

$$
L_{2}^{2}=k_{2}^{2}\left[\tilde{k}_{1}^{2}+(\xi-1) \frac{\tilde{k}_{1}^{2}(p-2)-2 V_{0}}{p-2+z-\theta}\right]^{-1},
$$

where $L_{2}$ is determined by $\tilde{k}_{1}$ and $k_{2}$ for an ansatz (2.25).

For given action parameters $\left(\delta, \lambda_{1}, \lambda_{2}, \gamma\left(\delta, \lambda_{1}, \lambda_{2}\right), V_{0}\right)$ and the solution parameter $\tilde{k}_{1}$, all the other solution parameters $\left(z, \theta, \xi, \zeta(\theta, \xi), \varphi_{0}, L_{r}, a_{0}, \tilde{k}_{2}\right)$ are fixed. Thus the total number of free parameters in the solution are four, which may be taken as $\left(z, \theta, \xi, \tilde{k}_{1}\right)$. Considering all the conditions (2.17)-(2.20), we classify the allowed parameter space in table 1 . For given $z$ and $\theta, \tilde{k}_{1}$ should be chosen to satisfy the inequality in the last column. To get some intuition on the content of table 1 it is useful to make figures representing the typical parameter ranges. Figure 1 shows five prototypical cases: $\xi=-1,0,0.5,1,2$ for $p=3$. Let us start with the case $\xi=1$ (figure $1(\mathrm{~d})$ ), where there are two regions: a rectangle $(z<0)$ and a pentagon $(z>1)$ of which upper left corner is a line not a curve. As $\xi$ increases (figure $1(\mathrm{e}))$ the pentagon moves to the right $(z>\xi)$ and its upper left corner becomes a curve while the rectangle moves to the up $(\theta>2 \xi)$. As $\xi$ decreases (figure 1(c,b)) the pentagon, of which upper left corner becomes curve, goes down and the rectangle does not move. After the pentagon becomes a rectangle at $\xi=0$ it keeps going down while the rectangle in $z<0$ starts moving to the left $(z<\xi)$. For comparison we collect the boundaries of five cases in figure $1(\mathrm{f})$.

\subsubsection{Class II: irrelevant charge}

The irrelevant charge means $a_{0}=0$ in the leading order so we start with an ansatz

$$
\begin{aligned}
\mathrm{d} s^{2} & =r^{\frac{2 \theta}{p-1}}\left[-\frac{\mathrm{d} t^{2}}{r^{2 z}}+\frac{L_{r}^{2} \mathrm{~d} r^{2}}{r^{2}}+\frac{\sum_{i=1}^{p-2} \mathrm{~d} \tilde{x}_{i}^{2}}{r^{2}}+\frac{\mathrm{d} \tilde{y}^{2}}{r^{2 \xi}}\right], \\
\varphi & =\varphi_{0} \log r, \quad A=0, \quad \chi_{i}=\tilde{k}_{1} \tilde{x}_{i}, \quad \chi_{p-1}=\tilde{k}_{2} \tilde{y},
\end{aligned}
$$

where $\varphi_{0}, \tilde{k}_{1}$ and $\tilde{k}_{2}$ are nonzero. 


\begin{tabular}{|clc|}
\hline \multicolumn{3}{|c|}{$\xi \leq 0$} \\
\hline $\begin{array}{c}|c| \\
1<z\end{array}$ & $\theta>p-1$ & $\zeta=2-p+\theta-\xi$ \\
& $\theta<\xi(p-1)$ & $\zeta=2-p+\theta-\xi$ \\
\hline$z<0$ & $\theta>p-1$ & $\zeta=2-p+\theta-\xi$ \\
$1<z \leq 1+\xi$ & $\theta<\frac{p-1}{2}\left(z-\sqrt{\left.(z-2)^{2}+\frac{4(\xi-1)(-z+1+\xi)}{p-1}\right)}\right)$ & $\zeta=2-p+\theta-\xi$ \\
$1+\xi<z$ & $\theta<\xi(p-1)$ & $\zeta=2-p+\theta-\xi$ \\
\hline & \multicolumn{1}{c}{$1 \leq \xi$} & \\
\hline$\xi<0$ & $\theta>\xi(p-1)$ & $\zeta=2-p+\theta-\xi$ \\
$1+\xi<z$ & $\theta<\frac{p-1}{2}\left(z-\sqrt{(z-2)^{2}+\frac{4(\xi-1)(-z+1+\xi)}{p-1}}\right)$ & $\zeta=2-p+\theta-\xi$ \\
For all cases: & $\theta<p-1$ & $\zeta=2-p+\theta-\xi$ \\
\hline
\end{tabular}

Table 1. Parameter range: Class I, $V_{0}>0$.

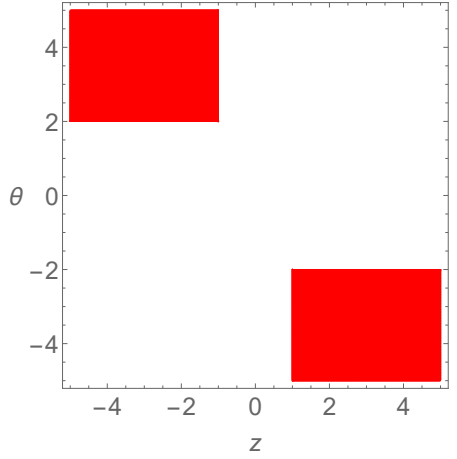

(a) $\xi=-1$

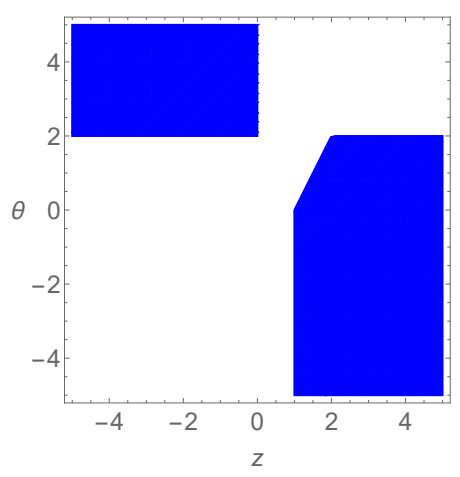

(d) $\xi=1$

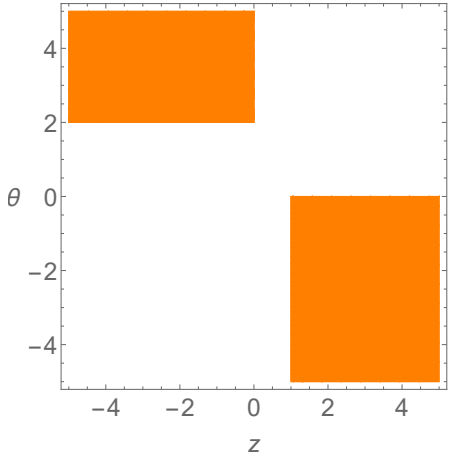

(b) $\xi=0$

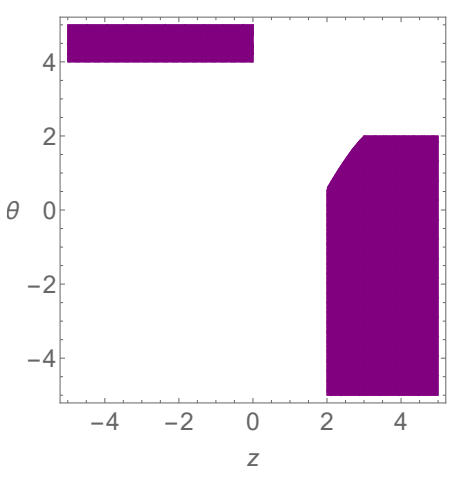

(e) $\xi=2$

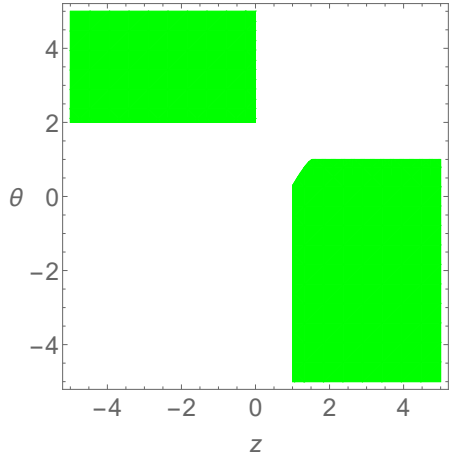

(c) $\xi=\frac{1}{2}$

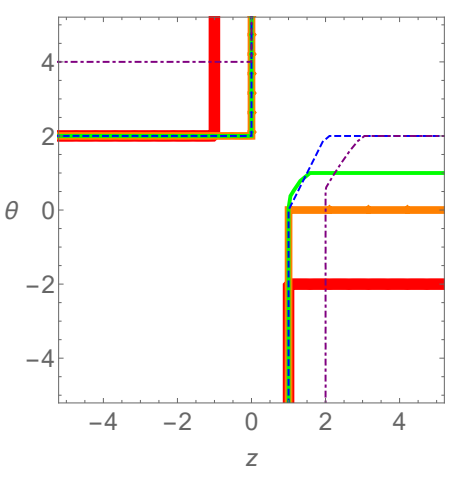

(f) The boundaries of (a)-(e)

Figure 1. Prototypical cases of table $1(p=3)$. 
This case corresponds to $a_{0}=0$ in class I so $\zeta$ does not appear in the leading order solution but will be introduced when we consider a subleading order. First, the 'exponent' solution-parameters $(z, \xi, \theta)$ may be expressed in terms of action-parameters $\left(\delta, \lambda_{1}, \lambda_{2}\right)$ as

$$
\begin{aligned}
& z=\frac{2-(p-1) \delta^{2}+(p-2) \lambda_{1}^{2}+\lambda_{2}^{2}}{\lambda_{1}\left((p-1) \delta+(p-2) \lambda_{1}+\lambda_{2}\right)}, \\
& \theta=-\frac{(p-1) \delta}{\lambda_{1}}, \quad \xi=\frac{\lambda_{2}}{\lambda_{1}},
\end{aligned}
$$

which is the same as the class I (2.28) except that $\zeta$ is undetermined. It is related to the non-existence of the constraints (2.30) and (2.29) in class II. Consequently, the action parameter $\gamma$ is free and $\left(\delta, \lambda_{1}, \lambda_{2}\right)$ may be written in terms of three solution-parameters $(z, \theta, \xi)$ :

$$
\delta=\frac{2 \theta}{(p-1) \varphi_{0}}, \quad \lambda_{1}=\frac{-2}{\varphi_{0}}, \quad \lambda_{2}=\frac{-2 \xi}{\varphi_{0}}
$$

where

$$
\varphi_{0}^{2}=\frac{2(p-1-\theta)(1+p(z-1)-z-\theta)}{p-1}-2(\xi-1)(\xi-z+1) .
$$

The coefficient parameters read

$$
\begin{aligned}
\varphi_{0} & =-\frac{2}{\lambda_{1}}, \\
L_{r}^{2} & =\frac{(2-p+\theta-\xi-z)(\theta-(p-1) z)}{V_{0}}, \\
\tilde{k}_{1}^{2} & =\frac{2 V_{0}(1-z)}{\theta-(p-1) z}, \quad \tilde{k}_{2}^{2}=\frac{2 V_{0}(\xi-z)}{\theta-(p-1) z} .
\end{aligned}
$$

Note that the last equations can be written as

$$
L_{1}^{2}=\frac{\theta-(p-1) z}{2 V_{0}(1-z)} k_{1}^{2}, \quad L_{2}^{2}=\frac{\theta-(p-1) z}{2 V_{0}(\xi-z)} k_{2}^{2},
$$

where $L_{1}$ and $L_{2}$ are determined by $k_{1}$ and $k_{2}$ for an ansatz (2.25).

After turning on the subleading gauge field mode generating a constant electric flux proportional to $a_{0}$

$$
A_{t}(r)=a_{0} r^{\zeta-z}
$$

we find

$$
\begin{aligned}
\zeta & =p-2-\frac{p-3}{p-1} \theta-\gamma \varphi_{0}+\xi \\
\varphi_{0}^{2} & =\frac{2(p-1-\theta)(1+p(z-1)-z-\theta)}{p-1}-2(\xi-1)(\xi-z+1),
\end{aligned}
$$

where $\zeta$ is a function of a free action parameter $\gamma$. This gauge field mode backreacts on metric and $\varphi$ at quadratic order as

$$
\sim r^{\beta}, \quad \text { where } \beta:=p-2+\zeta-\theta+\xi,
$$




\begin{tabular}{|c|c|c|}
\hline \multicolumn{3}{|c|}{$\xi \leq 0$} \\
\hline$z<\xi$ & $\theta>p-1$ & $\zeta>2-p+\theta-\xi$ \\
\hline $1<z$ & $\theta<\xi(p-1)$ & $\zeta<2-p+\theta-\xi$ \\
\hline \multicolumn{3}{|c|}{$0<\xi<1$} \\
\hline$z<0$ & $\theta>p-1$ & $\zeta>2-p+\theta-\xi$ \\
\hline $1<z \leq 1+\xi$ & $\theta<\frac{p-1}{2}\left(z-\sqrt{(z-2)^{2}+\frac{4(\xi-1)(-z+1+\xi)}{p-1}}\right)$ & $\zeta<2-p+\theta-\xi$ \\
\hline $1+\xi<z$ & $\theta<\xi(p-1)$ & $\zeta<2-p+\theta-\xi$ \\
\hline \multicolumn{3}{|c|}{$1 \leq \xi$} \\
\hline$z<0$ & $\theta>\xi(p-1)$ & $\zeta>2-p+\theta-\xi$ \\
\hline$\xi<z \leq 1+\xi$ & $\theta<\frac{p-1}{2}\left(z-\sqrt{(z-2)^{2}+\frac{4(\xi-1)(-z+1+\xi)}{p-1}}\right)$ & $\zeta<2-p+\theta-\xi$ \\
\hline $1+\xi<z$ & $\theta<p-1$ & $\zeta<2-p+\theta-\xi$ \\
\hline
\end{tabular}

Table 2. Parameter range: Class II, $V_{0}>0$.

which gives a constraint on $\zeta$ because $\beta$ should be positive(negative) if the IR is at $r \rightarrow 0(\infty)$. This constraint (inequality) was summarized in the third column in table 2 . After considering all conditions (2.17)-(2.20), we find that the parameter space of $z, \theta, \xi$ are the same as class I as shown in table 2. Therefore, figure 1 are valid also for class 2 . For given $z$ and $\theta, \gamma$ should be chosen to satisfy the inequality in the last column because $\zeta$ is a function of $\gamma$ for given $z, \theta$ and $\xi(2.46)$.

\subsection{The other classes}

In this subsection we consider the other possible classes explained in subsection 2.1. Similarly to the Class I and Class II in subsection 2.2, we first need to figure out the relations between solution-parameters and action-paramters class by class. Next, we need to consider the sub-leading modes to check the stability of the solution. Based on these information, finally, we can find the allowed parameter range by investigating the conditions (2.17)(2.21) and stability conditions of subleading modes. Here, we summarized only the allowed parameter range, relegating all details including the relations between solution-parameters and action-paramters to appendix A and B.

\subsubsection{Irrelevant axion: class III and VI}

Irrelevant axion means that $\tilde{k}_{1}=\tilde{k}_{2}=0\left(\chi_{i}=\chi_{p-1}=0\right)$ at leading order in the IR. In principle, there may be anisotropic solutions generated by the subleading axion mode due to anisotropy in the action, $\lambda_{1} \neq \lambda_{2}$. However, after turning on the subleading axion mode $\left(\chi_{i}=\tilde{k}_{1} \tilde{x}_{i}, \chi_{p-1}=\tilde{k}_{2} \tilde{y}\right)$ we find that $\lambda_{1}$ must be the same as $\lambda_{2}$ to satisfy the equations of motion in the subleading order. This does not mean that this case becomes the isotropic case. Because $\tilde{k}_{1} \neq \tilde{k}_{2}$ in the sub-leading order, it is a new kind of anisotropic solution with $\lambda_{1}=\lambda_{2}$. 
The irrelevant axion means $\tilde{k}_{1}=\tilde{k}_{2}=0$ in the leading order so we start with an ansatz

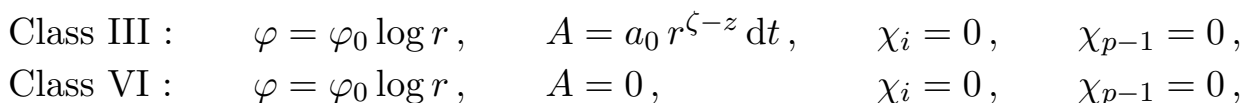

where $\varphi_{0}$ and $a_{0}$ are nonzero. The metric ansatz is the same as (2.13) with IR couplings (2.12).

Class III. After considering all conditions (2.17)-(2.20) and (A.9), we find that the allowed parameter space is

$$
\begin{array}{rll}
z<0, & \theta>p-1, & \varphi_{0} \lambda>-2, \\
1<z \leq 2, & \theta<(z-1)(p-1), & \varphi_{0} \lambda<-2, \\
2<z, & \theta<p-1, & \varphi_{0} \lambda<-2,
\end{array}
$$

which is the same as the case I for $\xi=1$ and represented in figure $1(\mathrm{~d})$. The last inequality and the extra condition for $\varphi_{0}$ are explained in (A.8)-(A.10) in appendix.

Class VI. After considering all conditions (2.17)-(2.20) with (A.17) and (A.18), we find that the parameter space is

$$
z=1, \quad \theta<0, \quad \zeta<\theta+1-p, \quad \varphi_{0} \lambda<-2 .
$$

\subsubsection{Mixed axions}

Here we consider the case that only one of the $\tilde{k}_{i}$ is nonzero, which we called 'mixed axions' in subsection 2.1 because the axion in one direction is marginally relevant and the axion in the other direction is irrelevant. This is a hybrid of the class I and III $\left(a_{0} \neq 0\right)$; and the class II and IV $\left(a_{0}=0\right)$. The class I-i and II-i means $\tilde{k}_{1}$ is nonzero and the class I-ii and II-ii means $\tilde{k}_{2}$ is nonzero. i.e. we assume that the leading solutions are written as

$$
\begin{array}{lllll}
\text { Class I-i : } & \varphi=\varphi_{0} \log r, & A=a_{0} r^{\zeta-z} \mathrm{~d} t, & \chi_{i}=\tilde{k}_{1} \tilde{x}_{i}, & \chi_{p-1}=0, \\
\text { Class I-ii : } & \varphi=\varphi_{0} \log r, & A=a_{0} r^{\zeta-z} \mathrm{~d} t, & \chi_{i}=0, & \chi_{p-1}=\tilde{k}_{2} \tilde{y}, \\
\text { Class II-i : } & \varphi=\varphi_{0} \log r, & A=0, & \chi_{i}=\tilde{k}_{1} \tilde{x}_{i}, & \chi_{p-1}=0 \\
\text { Class II-ii : } & \varphi=\varphi_{0} \log r, & A=0, & \chi_{i}=0, & \chi_{p-1}=\tilde{k}_{2} \tilde{y}
\end{array}
$$

where $\varphi_{0}$ and $a_{0}$ are nonzero. The metric ansatz is the same as (2.13) with IR couplings (2.12). The allowed parameter range is summarized in table $3,4,5$ and 6 respectively.

\section{$3 \quad$ Thermal diffusion and butterfly velocity}

In this section we consider diffusion in the anisotropic system. Diffusion in strongly correlated systems is a very interesting subject because of its proposed relation to the chaos properties such as the Lyapunov time $\left(\tau_{L}\right)$ and the butterfly velocity $\left(v_{B}\right)$, which are introduced in (1.2). At finite density, charge and energy diffusion are coupled and two diffusion 


\begin{tabular}{|c|c|c|c|c|}
\hline \multicolumn{5}{|c|}{$\xi \leq 0$} \\
\hline$z<\xi$ & $\theta>p-1$ & & $\zeta=2-p+\theta-\xi$ & $\frac{\lambda_{2}}{\lambda_{1}}<\xi$ \\
\hline \multicolumn{5}{|c|}{$0<\xi<1$} \\
\hline$z<0$ & $\theta>p-1$ & & $\zeta=2-p+\theta-\xi$ & $\frac{\lambda_{2}}{\lambda_{1}}<\xi$ \\
\hline \multicolumn{5}{|c|}{$1 \leq \xi$} \\
\hline$\xi<z \leq 1+\xi$ & $\theta<\frac{p-1}{2}$ & $\left.\sqrt{(z-2)^{2}+\frac{4(\xi-1)(-z+1+\xi)}{p-1}}\right)$ & $\zeta=2-p+\theta-\xi$ & $\frac{\lambda_{2}}{\lambda_{1}}>\xi$ \\
\hline $1+\xi<z$ & $\theta<p-1$ & & $\zeta=2-p+\theta-\xi$ & $\frac{\lambda_{2}}{\lambda_{1}}>\xi$ \\
\hline
\end{tabular}

Table 3. Parameter range: Class I-i, $V_{0}>0$.

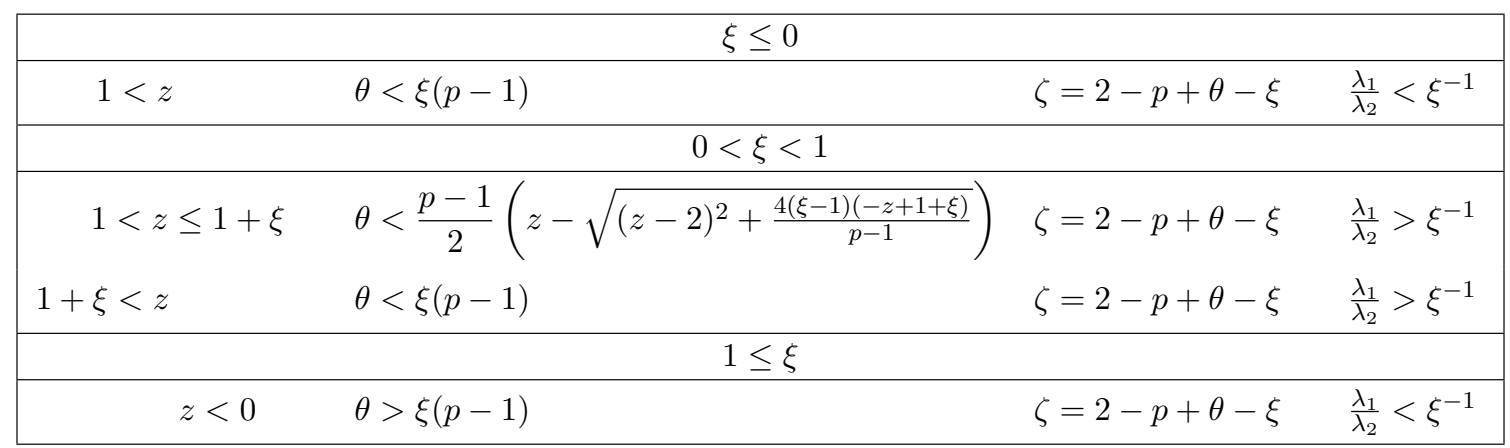

Table 4. Parameter range: Class I-ii, $V_{0}>0$.

\begin{tabular}{|cccc|}
\hline \multicolumn{4}{|c|}{$\xi<0$} \\
\hline$z=\xi$ & $\theta>p-1$ & $\zeta>2-p+\theta-\xi$ & $\frac{\lambda_{2}}{\lambda_{1}}<\xi$ \\
\hline \multicolumn{4}{|c|}{$1<\xi$} \\
\hline$z=\xi$ & $\theta<\frac{p-1}{2}\left(\xi-\sqrt{(\xi-2)^{2}+\frac{4(\xi-1)}{p-1}}\right)$ & $\zeta<2-p+\theta-\xi$ & $\frac{\lambda_{2}}{\lambda_{1}}>\xi$ \\
\hline
\end{tabular}

Table 5. Parameter range: Class II-i, $V_{0}>0$.

constants $D_{ \pm}$describing the coupled diffusion of charge and energy can be obtained by the generalized Einstein relation $[10]^{10}$

$$
\begin{aligned}
D_{+} D_{-} & =\frac{\sigma}{\chi} \frac{\kappa}{c_{\rho}}, \\
D_{+}+D_{-} & =\frac{\sigma}{\chi}+\frac{\kappa}{c_{\rho}}+\frac{T(\tilde{\zeta} \sigma-\chi \alpha)^{2}}{c_{\rho} \chi^{2} \sigma},
\end{aligned}
$$

where $\sigma, \alpha$, and $\kappa$ are the electric, thermoelectric and thermal conductivity respectively. $\chi$ is the compressibility, $c_{\rho}$ is the specific heat at fixed charge density and $\tilde{\zeta}$ is the thermoelectric susceptibility.

If the charge density is zero, since $\alpha=\tilde{\zeta}=0$, the 'mixing term' (the third term in (3.2)) vanishes. In this case $D_{ \pm}$are decoupled and $D_{+}$and $D_{-}$can be identified with

\footnotetext{
${ }^{10}$ The conductivities may be diagonalized as in [57].
} 


\begin{tabular}{|lccc|}
\hline \multicolumn{4}{|c|}{$\xi \leq 0$} \\
\hline$z=1$ & $\theta<\xi(p-1)$ & $\zeta<2-p+\theta-\xi$ & $\frac{\lambda_{1}}{\lambda_{2}}<\xi^{-1}$ \\
\hline \multicolumn{4}{|c|}{$0<\xi<1$} \\
\hline$z=1$ & $\theta<\frac{p-1}{2}\left(1-\sqrt{1+\frac{4(\xi-1) \xi}{p-1}}\right)$ & $\zeta<2-p+\theta-\xi$ & $\frac{\lambda_{1}}{\lambda_{2}}>\xi^{-1}$ \\
\hline
\end{tabular}

Table 6. Parameter range: Class II-ii, $V_{0}>0$.

the charge diffusivity $\left(D_{C}\right)$ and the thermal diffusivity $\left(D_{T}\right)$ respectively. The mixing term is also negligible in the incoherent regime where momentum relaxations is strong $\left(k_{i} / \mu \gg 1, k_{i} / T \gg 1\right)[16]$. Furthermore, It has been shown that in the low temperature limit of the scaling geometry studied in section 2, the mixing term is negligible [34]. ${ }^{11} \mathrm{In}$ this section, we consider this low temperature limit and focus on the anisotropic thermal diffusivities defined by

$$
D_{T, x}:=\frac{\kappa_{x x}}{c_{\rho}}, \quad D_{T, y}:=\frac{\kappa_{y y}}{c_{\rho}},
$$

and a specific combinations:

$$
\mathbb{E}_{x}:=\frac{D_{T, x}}{v_{B, x}^{2} \tau_{L}}, \quad \mathbb{E}_{y}:=\frac{D_{T, y}}{v_{B, y}^{2} \tau_{L}}
$$

where $v_{B, i}$ is the butterfly velocity in the $i$ direction.

The essential idea of the following analysis was already described in [34] and we closely follow the steps therein. Our goal here is to extend the results in [34] in three aspects. i) to understand $\mathbb{E}_{x}$ and $\mathbb{E}_{y}$ in terms of scaling exponents $z$ and $\xi$ and ii) to identify the allowed range of $\mathbb{E}_{x}$ and $\mathbb{E}_{y}$ and see if there is any universal lower or upper bound. iii) to extend the formalism to the case where $g_{t t} \neq g_{r r}^{-1}$. To achieve our goals, the analysis in section 2 are necessary.

For simplicity let us consider $p=3$, in which case the action (2.1) becomes

$$
S=\int \mathrm{d}^{4} x \sqrt{-g}\left[R-\frac{1}{2}(\partial \varphi)^{2}+V(\varphi)-\frac{1}{4} Z(\varphi) F^{2}-\frac{1}{2} W_{1}(\varphi)\left(\partial \chi_{1}\right)^{2}-\frac{1}{2} W_{2}(\varphi)\left(\partial \chi_{2}\right)^{2}\right] .
$$

We consider a general metric solution of the form

$$
\mathrm{d} s^{2}=-D(r) \mathrm{d} t^{2}+B(r) \mathrm{d} r^{2}+C_{1}(r) \mathrm{d} x^{2}+C_{2}(r) \mathrm{d} y^{2},
$$

where we allow $B \neq D$ and slightly generalize the formulas in [34] to the case $B \neq D^{-1}$.

\footnotetext{
${ }^{11}$ The suppression of the mixing term in the low temperature limit of the scaling geometry has been explained in detail in appendix A of [34]. The only modification in our case is the effect of anisotropic parameter $\xi$. Because the argument in [34] is also valid in the anisotropic case, here we report the change of key formula (A.8) in [34] due to the anisotropic parameter $\xi$ :

$$
\frac{T}{c_{\rho}}\left(\frac{\alpha_{i i}}{\sigma_{i i}}-\left(\frac{\partial s}{\partial \rho}\right)_{T}\right)^{2} \sim T^{1+((p-2)+\xi-\theta) / z},
$$

where $i i$ denotes $x x$ or $y y$ and we used $c_{\rho} \sim(\partial s / \partial \rho)_{T} \sim T^{((p-2)+\xi-\theta) / z}$ and $\alpha_{i i} / \sigma_{i i} \sim T^{((p-2)+\xi-\theta) / z}$.
} 
The IR asymptotic form of the metric (3.6) is assumed to be (2.13), while its UV asymptotic form is supposed to be isotropic $\mathrm{AdS}_{4}$ space. Note that the couplings and potentials (2.12) are valid only at IR. For the asymptotically $\mathrm{AdS}_{p+1}$ space at UV, we need to have suitable asymptotic potential terms at UV: it will be at least necessary to have $V(\varphi)$ at $\mathrm{UV}$ such that

$$
V(0)=-\frac{p(p-1)}{L^{2}}, \quad V^{\prime}(0)=0, \quad V^{\prime \prime}(0)=m^{2},
$$

where we set $\varphi=0$ at UV and $m^{2}$ (the scalar mass squared) should not violate the BF (Breitenlohner-Freedman) bound of a scalar field in $\mathrm{AdS}_{p+1}$ space. For example, we may consider the following form:

$$
V(\varphi)=a_{1} e^{b_{1} \varphi}+a_{2} e^{b_{2} \varphi}+\cdots
$$

and choose the parameters $a_{1}, a_{2}, b_{1}$, and $b_{2}$ to satisfy the constraints (3.7), which can be done always in principle. For more details, we refer to [58].

From the metric (3.6) the temperature and entropy density read

$$
T=\left.\frac{1}{4 \pi} \frac{\left|D^{\prime}\right|}{\sqrt{D B}}\right|_{r_{h}}, \quad s=\left.4 \pi \sqrt{C_{1} C_{2}}\right|_{r_{h}} .
$$

The electric conductivity $\left(\sigma_{x x}\right)$, thermoelectric conductivity $\left(\alpha_{x x}\right)$, and thermal conductivity $\left(\bar{\kappa}_{x x}\right)$ have been obtained in terms of horizon data in [59]. In our convention they read ${ }^{12}$

$$
\sigma_{x x}=\left.\left(\frac{\rho^{2}}{k_{1}^{2} W_{1} \sqrt{C_{2} C_{1}}}+Z \sqrt{\frac{C_{2}}{C_{1}}}\right)\right|_{r_{h}}, \quad \alpha_{x x}=\left.\frac{4 \pi \rho}{k_{1}^{2} W_{1}}\right|_{r_{h}}, \quad \bar{\kappa}_{x x}=\left.\frac{4 \pi s T}{k_{1}^{2} W_{1}}\right|_{r_{h}},
$$

where $\rho$ is the charge density

$$
\rho=\sqrt{\frac{C_{1} C_{2}}{D B}} Z A_{t}^{\prime}
$$

which is easily seen in (2.10). The thermal conductivity with an open circuit condition $\left(\kappa_{x x}\right)$ is

$$
\kappa_{x x}=\bar{\kappa}_{x x}-\frac{T \alpha_{x x}^{2}}{\sigma_{x x}}=\left.\frac{4 \pi s T Z(\varphi(r)) C_{2}(r)}{\rho^{2}+k_{1}^{2} W_{1}(\varphi(r)) Z(\varphi(r)) C_{2}(r)}\right|_{r_{h}} .
$$

All conductivities in (3.10) and (3.12) depend on the metric, the forms of couplings and the profiles of the matter fields. However, the key observation made in [34] is that the thermal conductivity with an open ciruit condition $\left(\kappa_{x x}\right)$ is a function only of the metric. It can be seen from the Einstein equations. By eliminating the second term including $V$ in (2.6) and (2.8) we have

$$
\begin{aligned}
\frac{B^{\prime} C_{1}^{\prime}}{B^{2}} & +\frac{C_{1}^{\prime 2}}{B C_{1}}-\frac{C_{1}^{\prime} C_{2}^{\prime}}{B C_{2}}-\frac{C_{1} B^{\prime} D^{\prime}}{B^{2} D}+\frac{C_{1} C_{2}^{\prime} D^{\prime}}{B C_{2} D}-\frac{C_{1} D^{\prime 2}}{B D^{2}}-\frac{2 C_{1}^{\prime \prime}}{B}+\frac{2 C_{1} D^{\prime \prime}}{B D} \\
& =2 k_{1}^{2} W_{1}+\frac{2 Z C_{1} A_{t}^{\prime 2}}{B D} .
\end{aligned}
$$

\footnotetext{
${ }^{12}$ These DC formulas have been confirmed by computing the optical conductivities and taking the zero frequency limit [60-62]. See also [63, 64] which were the first papers developing the techniques to calculate the electric conductivity in terms of the black hole horizon data in massive gravity.
} 
Note that the right hand side is a combination of the stress energy tensor in the Einstein equations and it is the combination that appears in the denominator of (3.12) after substituting $\rho$ with (3.11). Thus, $\kappa_{x x}$ can be expressed only in terms of metric:

$$
\kappa_{x x}=\left.\frac{32 \pi^{2} T\left(C_{1}^{-1} C_{2}\right)^{\frac{3}{2}} D^{\prime}}{\left[\left(C_{1}^{-1} C_{2}\right)(B D)^{-1} D^{\prime 2}\right]^{\prime}}\right|_{r_{h}} .
$$

Note that the dependence of $\kappa_{x x}$ on the matter fields and the couplings are still implicitly encoded in the metric but there is no explicit matter and coupling dependence in $\kappa_{x x}$. This suggests that there may be some universal feature. For the conductivities in the $y$ direction, we only need to replace the subscripts $x \rightarrow y$ and $1 \leftrightarrow 2$ from (3.10) and (3.14). For exmaple, from (3.14)

$$
\kappa_{y y}=\left.\frac{32 \pi^{2} T\left(C_{2}^{-1} C_{1}\right)^{\frac{3}{2}} D^{\prime}}{\left[\left(C_{2}^{-1} C_{1}\right)(B D)^{-1} D^{\prime 2}\right]^{\prime}}\right|_{r_{h}} .
$$

The formula (3.14) and (3.15) are reduced to the ones in [34] if $B=D^{-1}$.

The specific heat can be computed from the entropy density in (3.9) as

$$
c_{\rho}=T\left(\frac{\partial s}{\partial T}\right)_{\rho}=\left.\frac{2 \pi T\left(C_{1} C_{2}\right)^{\prime}}{\sqrt{C_{1} C_{2}}}\right|_{r_{h}} \frac{\partial r_{h}}{\partial T},
$$

where $r_{h}$ is a function of $T$ obtained by the first equation of (3.9). In principle, $\frac{\partial r_{h}}{\partial T}$ also can be written in terms of the derivatives of the metric but it is not so illuminating.

Finally, the butterfly velocities can be computed holographically by considering a shock wave geometry and they are written in terms of the metric data at horizon [22, 34]. For anisotropic case

$$
v_{B, x}=\left.\frac{2 \pi T}{\sqrt{C_{1}} m}\right|_{r_{h}}, \quad v_{B, y}=\left.\frac{2 \pi T}{\sqrt{C_{2}} m}\right|_{r_{h}}
$$

where

$$
m=\sqrt{\left.\pi T\left(\frac{\left(C_{1} C_{2}\right)^{\prime}}{C_{1} C_{2} \sqrt{B D}}\right)\right|_{r_{h}}},
$$

which slightly generalize the formula of $[22,34]$ to the case $B \neq D^{-1}$.

Having the general formulas for the thermal conductivity $\left(\kappa_{x x}, \kappa_{y y}\right)$, specific heat $\left(c_{\rho}\right)$, and the butterfly velocity $\left(v_{B, x}, v_{B, y}\right)$ for a metric of the form (3.6), we turn to our anisotropic model in section 2. For all classes considered in there the metric is of the form

$$
\mathrm{d} s^{2}=r^{\theta}\left(-f(r) \frac{\mathrm{d} t^{2}}{r^{2 z}}+\frac{L_{r}^{2} \mathrm{~d} r^{2}}{f(r) r^{2}}+\frac{L_{1}^{2} \mathrm{~d} x^{2}}{r^{2}}+\frac{L_{2}^{2} \mathrm{~d} y^{2}}{r^{2 \xi}}\right)
$$

with the emblackening factor

$$
f(r)=1-\left(\frac{r}{r_{h}}\right)^{z+1-\theta+\xi}
$$


The temperature (3.9) is related to the horizon position $r_{h}$ as follows.

$$
T=\frac{r_{h}^{-z}|1+z-\theta+\xi|}{4 \pi L_{r}}, \quad r_{h}=\left(\frac{|1+z-\theta+\xi|}{4 \pi L_{r} T}\right)^{\frac{1}{z}}
$$

Thermal conductivities (3.14) and (3.15) are

$$
\kappa_{x x}=\frac{4 \pi}{2(1-z)} L_{r} L_{1}^{-1} L_{2} r_{h}^{\theta-z-\xi+1}, \quad \kappa_{y y}=\frac{4 \pi}{2(\xi-z)} L_{r} L_{1} L_{2}^{-1} r_{h}^{\theta-z+\xi-1}
$$

and the specific heat (3.16) is computed as

$$
c_{\rho}=T\left(\frac{\partial s}{\partial T}\right)_{\rho}=4 \pi \frac{1+\xi-\theta}{z} L_{1} L_{2} r_{h}^{\theta-1-\xi}
$$

from the entropy density (3.9)

$$
s=4 \pi L_{1} L_{2} r_{h}^{\theta-(1+\xi)} \sim T^{\frac{1+\xi-\theta}{z}} .
$$

In (3.22) and (3.23) we replaced $T$ with $r_{h}$ by using (3.21) to simplify the expression.

Thus, the diffusivities are

$$
\begin{aligned}
& D_{T, x}=\frac{\kappa_{x x}}{c_{\rho}}=\frac{z}{(2 z-2)(\theta-1-\xi)} L_{r} L_{1}^{-2} r_{h}^{2-z}, \\
& D_{T, y}=\frac{\kappa_{y y}}{c_{\rho}}=\frac{z}{(2 z-2(2-\xi))(\theta-1-\xi)} L_{r} L_{2}^{-2} r_{h}^{2 \xi-z},
\end{aligned}
$$

and the butterfly velocities (3.17) are

$$
v_{B, x}^{2}=\frac{2 \pi T}{\theta-1-\xi} L_{r} L_{1}^{-2} r_{h}^{2-z}, \quad v_{B, y}^{2}=\frac{2 \pi T}{\theta-1-\xi} L_{r} L_{2}^{-2} r_{h}^{2 \xi-z}
$$

Finally, by noticing that $\tau_{L}=(2 \pi T)^{-1}$ we have

$$
\begin{aligned}
& \mathbb{E}_{x}=\frac{D_{T, x}}{v_{B, x}^{2} \tau_{L}}=\frac{1}{2} \frac{z_{x}}{z_{x}-1}=\frac{1}{2} \frac{z}{z-1}, \\
& \mathbb{E}_{y}=\frac{D_{T, y}}{v_{B, y}^{2} \tau_{L}}=\frac{1}{2} \frac{z_{y}}{z_{y}-1}=\frac{1}{2} \frac{z}{z-\xi} .
\end{aligned}
$$

Notice that the $\mathbb{E}_{x}$ and $\mathbb{E}_{y}$ depend only on $z$ and $\xi$ irrespective of $\theta$ and $\zeta$. They are also independent of charge density $\rho$ and momentum relaxations $k_{1}$ and $k_{2}$. This universality is nontrivial because the thermal conductivities, specific heat and butterfly velocity, all of them depend on $\left(\theta, \zeta, \rho, k_{1}, k_{2}\right)$ through $\left(L_{r}, L_{1}, L_{2}, r_{h}\right)$. When it comes to the combinations $\mathbb{E}_{x}$ and $\mathbb{E}_{y}$, all $L_{r}, L_{1}, L_{2}$ and $r_{h}$ are canceled out. 
To investigate if there is any lower or upper bound of $\mathbb{E}_{x}$ and $\mathbb{E}_{y}$, we need to understand the parameter region of $z$ and $\xi$. We will restrict ourselves to positive $z_{i}$. Based on the allowed parameter region obtained in section 2 we find

- Class I and II

$$
\begin{aligned}
& \frac{\lambda_{2}}{\lambda_{1}} \geq 1 \quad \Rightarrow \quad \frac{1}{2} \leq \mathbb{E}_{x}<\frac{1}{2}\left(\frac{1}{1-\xi^{-1}}\right), \quad \frac{1}{2} \leq \mathbb{E}_{y}, \\
& \frac{\lambda_{1}}{\lambda_{2}} \geq 1 \quad \Rightarrow \quad \frac{1}{2} \leq \mathbb{E}_{x}, \quad \frac{1}{2} \leq \mathbb{E}_{y}<\frac{1}{2}\left(\frac{1}{1-\xi}\right) \text {, }
\end{aligned}
$$

where $\xi=\frac{\lambda_{2}}{\lambda_{1}}$.

- Class I-i

$$
\frac{\lambda_{2}}{\lambda_{1}}>1 \Rightarrow \frac{1}{2} \leq \mathbb{E}_{x}<\frac{1}{2}\left(\frac{1}{1-\xi^{-1}}\right), \quad \frac{1}{2} \leq \mathbb{E}_{y},
$$

where $\xi=-\frac{\gamma+\delta+\lambda_{1}}{\lambda_{1}}$. Here $k_{2}=0$ and $\frac{\lambda_{1}}{\lambda_{2}}>1$ is not allowed.

- Class I-ii

$$
\frac{\lambda_{1}}{\lambda_{2}}>1 \Rightarrow \frac{1}{2} \leq \mathbb{E}_{x}, \quad \frac{1}{2} \leq \mathbb{E}_{y}<\frac{1}{2}\left(\frac{1}{1-\xi}\right)
$$

where $\xi=-\frac{\lambda_{2}}{\gamma+\delta+\lambda_{2}}$. Here $k_{1}=0$ and $\frac{\lambda_{2}}{\lambda_{1}}>1$ is not allowed.

- Class II-i

$$
\frac{\lambda_{2}}{\lambda_{1}}>1 \Rightarrow \frac{1}{2} \leq \mathbb{E}_{x}=\frac{1}{2}\left(\frac{1}{1-\xi^{-1}}\right)
$$

where $\xi=\frac{2-2 \delta^{2}+\lambda_{1}^{2}}{\lambda_{1}\left(2 \delta+\lambda_{1}\right)}$. Here $k_{2}=0$ and $\frac{\lambda_{1}}{\lambda_{2}}>1$ is not allowed. $z=\xi$ so $\mathbb{E}_{y}$ is not computed in our method.

- Class II-ii

$$
\frac{\lambda_{1}}{\lambda_{2}}>1 \Rightarrow \frac{1}{2} \leq \mathbb{E}_{y}=\frac{1}{2}\left(\frac{1}{1-\xi}\right),
$$

where $\xi=\frac{\lambda_{2}\left(2 \delta+\lambda_{2}\right)}{2-2 \delta^{2}+\lambda_{2}^{2}}$ with $z=1$. Here $k_{1}=0$ and $\lambda_{2} / \lambda_{1}>1$ is not allowed. $z=1$ so $\mathbb{E}_{x}$ is not computed in our method.

- Class III

$$
\frac{\lambda_{1}}{\lambda_{2}}=1 \quad \Rightarrow \quad \frac{1}{2} \leq \mathbb{E}_{x}=\mathbb{E}_{y}=\frac{1}{2}\left(\frac{z}{z-1}\right)
$$

- Class IV: $z=1$ so $\mathbb{E}_{i}$ cannot be computed in our method.

We find that the lower bound of $\mathbb{E}_{i}$ is always $1 / 2$. However, contrary to the isotropic case, there may be an upper bound for class I, II, I-i, and I-ii. All of these have at least one marginally relevant axion. This upper bound can be understood from the fact that for $0<\xi \leq 1, z>1$ and for $\xi \geq 1, z>\xi$. For example, in (3.29), even though $\mathbb{E}_{x}$ does not depend on $\xi$ explicitly, its range depends on $\xi$ because the available parameter range of $z$ depends on $\xi$. In this case it is $z>\xi$, which gives an upper bound. 


\section{Conclusion}

In this paper, we have studied the holographic systems so called 'Q-lattice' or EinsteinMaxwell-Dilaton theory coupled to 'Axion' fields (EMDA). The dilaton is introduced to support the scaling IR geometry and the axion fields are included to break translational symmetry. Our main focus is to study the effect of spatial anisotropy which is introduced in two ways: i) by making the different dilaton couplings to axion fields and ii) by considering the different momentum relaxation parameters for spatial directions. The former is characterized by $\lambda_{1}$ and $\lambda_{2}$ in (2.12) and the latter is done by $k_{1}$ and $k_{2}$ in (2.5).

First, we have extended four classes of the isotropic IR geometry [32] to the anisotropic case, which yields eight classes. For marginally relevant axion, where the momentum relaxation parameters $\left(k_{1}, k_{2}\right)$ appear explicitly in the leading IR solution, the anisotropy of $k_{i}$ and $\lambda_{i}$ are related $(i=1,2)$. However, for irrelevant axion, where the momentum relaxation parameters $\left(k_{1}, k_{2}\right)$ do not appear in the leading IR solution, the sub-leading order mode analysis imposes the conditions $\lambda_{1}=\lambda_{2}$, and $k_{i}$ and $\lambda_{i}$ are not related. It is also possible that only one of $k_{i}$ is zero. Therefore, in total there are four classes in terms of 'relevance' of axions: in the leading order solution, both $k_{i}$ are nonzero, both $k_{i}$ are zero, only one $k_{i}$ is zero (in $2+1$ field theory dimension, $k_{1}=0$ and $k_{2}=0$ are equivalent.) For every classes the charge may be marginally relevant or irrelevant. i.e. the temporal gauge filed $A_{t}$ may be non-zero or zero in the leading order solution. Therefore, we have eight classes in total.

The solutions have many parameters so called 'solution-parameters', which include two critical exponents: $z_{i}$ for a dynamical exponent along $i$-direction, $\theta$ for a hyperscaling violating exponent, and $\zeta$ for the anomalous dimension of the field theory charge density. In our representation $z_{x}=z$ and $z_{y}=z / \xi$ where $\xi$ characterizes the anisotropy between $x$ direction and $y$-direction. These solution-parameters should be restricted by some physical conditions such as reality, positive specific heat, and null energy conditions. We have identified those conditions in tables $1-10$ and figure 1.

Next, we have considered thermal diffusion in anisotropic cases. For the holographic systems with the metric

$$
\mathrm{d} s^{2}=-D(r) \mathrm{d} t^{2}+B(r) \mathrm{d} r^{2}+C_{1}(r) \mathrm{d} x^{2}+C_{2}(r) \mathrm{d} y^{2},
$$

the thermal conductivity, specific heat, butterfly velocity in $x$-direction can be computed in terms of the horizon data as

$$
\begin{aligned}
\kappa_{x x} & =\left.\frac{32 \pi^{2} T\left(C_{1}^{-1} C_{2}\right)^{\frac{3}{2}}\left|D^{\prime}\right|}{\left[\left(C_{1}^{-1} C_{2}\right)(B D)^{-1} D^{\prime 2}\right]^{\prime}}\right|_{r_{h}}, \\
c_{\rho} & =\left.\frac{2 \pi T\left(C_{1} C_{2}\right)^{\prime}}{\sqrt{C_{1} C_{2}}}\right|_{r_{h}} \frac{\partial r_{h}}{\partial T}, \quad v_{B, x}^{2}=\left.\frac{4 \pi T\left(C_{2} \sqrt{B D}\right)}{\left(C_{1} C_{2}\right)^{\prime}}\right|_{r_{h}},
\end{aligned}
$$

which give

$$
\mathbb{E}_{x}=\frac{D_{T, x}}{v_{B, x}^{2} \tau_{L}}=\left.\frac{8 \pi C_{1}^{-1} C_{2}\left|D^{\prime}\right|}{\sqrt{B D}\left[C_{1}^{-1} C_{2}(B D)^{-1} D^{\prime 2}\right]^{\prime}}\right|_{r_{h}} \frac{\partial T}{\partial r_{h}},
$$


where $\tau_{L}=(2 \pi T)^{-1}$ is the Lyapunov time. We may obtain the quantities in $y$-direction by switching the subscript $1 \leftrightarrow 2$. Notice that $\kappa_{x x}$ is originally a function of the couplings and the profiles of the matter fields. All these explicit matter dependences were replaced by the metric thanks to the Einstein equations, which suggests that there may be some universal feature. However, (4.4) is still a complicated function of the metric components so can not guarantee a universality by itself. For example, in general, it can be a function of $r_{h}$, which is a function of temperature, charge density, and momentum relaxations etc.

For the IR scaling geometry we have studied in section 2 the combination (4.4) is reduced to a simple universal form:

$$
\mathbb{E}_{x}=\frac{1}{2}\left(\frac{z_{x}}{z_{x}-1}\right)=\frac{1}{2}\left(\frac{z}{z-1}\right), \quad \mathbb{E}_{y}=\frac{1}{2}\left(\frac{z_{y}}{z_{y}-1}\right)=\frac{1}{2}\left(\frac{z}{z-\xi}\right) .
$$

Notice that in this geometry the parameters $\mathbb{E}_{x}$ and $\mathbb{E}_{y}$ depend only on $z$ and $\xi$ irrespective of $\theta, \zeta$, charge density $\rho$ and momentum relaxations $\left(k_{1}\right.$ and $\left.k_{2}\right)$. This universality is due to cancellations between three quantities $\left\{\kappa_{x x}\left(\kappa_{y y}\right), c_{\rho}, v_{B, x}\left(v_{B, y}\right)\right\}$, all of which depend on $\left\{\theta, \zeta, \rho, k_{1}, k_{2}\right\}$ as well as $\{z, \xi\}$.

We also studied the possible range of $\mathbb{E}_{x}$ and $\mathbb{E}_{y}$ to see if there is any universal lower or upper bound. Based on the parameter range analyzed in section 2 we find that the lower bound of $\mathbb{E}_{i}$ is always $1 / 2$. However, there may be an upper bound due to anisotropy, which was summarized in (3.29)-(3.35). It would be interesting to understand how much this lower and upper bound is robust in deformation of the theory, for example, with finite magnetic field $[34,65]$ or with higher derivative gravity in 'Q-lattice' models.

In holography, it might be possible to construct theories with a less (or non) universal $\mathbb{E}_{i}$ or without any universal bound of $\mathbb{E}_{i}$ by considering some complicated enough bulk models. In condensed matter systems, as pointed out in [34], the expression $\mathbb{E}_{i}$ in (4.5) is not expected to be universal for all systems with the same dynamical critical exponent. For example, some models with $z=3 / 2$ may give $\mathbb{E} \sim 0.42$ which is different from (4.5) [31]. Thus, a counter example regarding the universality in holographic models is not always bad. The important direction will be to classify the conditions for the universality and understand its origin from both gravity and condensed matter perspective, towards experimental understanding and applications.

\section{Acknowledgments}

We would like to thank Matteo Baggioli, Blaise Gouteraux, Ki-Seok Kim, Andrew Lucas, Sang-Jin Sin and Yunseok Seo for valuable discussions and correspondence. The work of D. Ahn, Y. Ahn, H.-S. Jeong, K.-Y. Kim and C. Niu was supported by Basic Science Research Program through the National Research Foundation of Korea(NRF) funded by the Ministry of Science, ICT \& Future Planning(NRF- 2017R1A2B4004810) and GIST Research Institute(GRI) grant funded by the GIST in 2017. The work of W.J. Li was supported by the Fundamental Research Funds for the Central Universities No. DUT $16 \mathrm{RC}(3) 097$ as well as NSFC Grants No. 11375026. We also would like to thank the APCTP(Asia-Pacific Center for Theoretical Physics) focus program, "Geometry and Holography for Quantum Criticality" in Pohang, Korea for the hospitality during our visit, where part of this work was done. 


\section{A Irrelevant axion}

Irrelevant axion means that $k_{1}=k_{2}=0\left(\chi_{i}=\chi_{p-1}=0\right)$ at leading order in the IR. Because $k_{1} \neq k_{2}$ in the sub-leading order, it is a new kind of anisotropic solution with $\lambda_{1}=\lambda_{2}$.

\section{A.1 Class III: marginally relevant charge}

The irrelevant axion means $\tilde{k}_{1}=\tilde{k}_{2}=0$ in the leading order so we start with an ansatz

$$
\begin{aligned}
\mathrm{d} s^{2} & =r^{\frac{2 \theta}{p-1}}\left[-\frac{\mathrm{d} t^{2}}{r^{2 z}}+\frac{L_{r}^{2} \mathrm{~d} r^{2}}{r^{2}}+\frac{\sum_{i=1}^{p-2} \mathrm{~d} \tilde{x}_{i}^{2}}{r^{2}}+\frac{\mathrm{d} \tilde{y}^{2}}{r^{2 \xi}}\right], \\
\varphi & =\varphi_{0} \log r, \quad A=a_{0} r^{\zeta-z} \mathrm{~d} t, \quad \chi_{i}=0, \quad \chi_{p-1}=0,
\end{aligned}
$$

where $\varphi_{0}$ and $a_{0}$ are nonzero.

By the equations of motion, solution-parameters $\left(z, \theta, \xi, \varphi_{0}, \zeta\right)$ may be expressed in terms of two action-parameters $(\delta, \gamma)$ as

$$
\begin{aligned}
& z=\frac{\varphi_{0}^{2}\left(\delta^{2}(p-1)-2\right)-4(p-1)}{2(p-1)\left(\delta \varphi_{0}-2\right)}, \quad \varphi_{0}=\frac{2(p-1)}{\gamma+\delta(p-2)}, \\
& \theta=\frac{(p-1) \delta \varphi_{0}}{2}, \quad \xi=1, \quad \zeta=\frac{(\delta-\gamma) \varphi_{0}}{2} .
\end{aligned}
$$

They satisfy the following constraint

$$
\zeta=-(p-1-\theta) \equiv-d_{\theta} \equiv \zeta_{I},
$$

which corresponds to the relation between action parameters

$$
\gamma=(2-p) \delta+\frac{2(p-1)}{\varphi_{0}}
$$

The remaining solutions parameters are

$$
\begin{aligned}
L_{r}^{2} & =\frac{(z+p-\theta-1)(z+p-\theta-2)}{V_{0}}, \\
a_{0}^{2} & =\frac{2(-1+z)}{-1+p+z-\theta},
\end{aligned}
$$

where $\zeta \neq z$ and $\zeta \neq z-1$ are assumed.

The action-parameters $(\delta, \gamma)$ may be written in terms of two solution-parameters $(z, \theta)$ :

$$
\delta=\frac{2 \theta}{(p-1) \varphi_{0}}, \quad \gamma=\frac{-2 \zeta+\frac{2}{p-1} \theta}{\varphi_{0}},
$$

where

$$
\begin{aligned}
\varphi_{0}^{2} & =2\left(\frac{\theta^{2}}{p-1}-z \zeta+1-p\right) \\
& =\frac{2(p-1-\theta)(1+p(z-1)-z-\theta)}{p-1} .
\end{aligned}
$$


Note that all formulas so far are independent of $\lambda_{1}$ and $\lambda_{2}$ and they are free. However, after introducing the subleading axion mode $\left(\chi_{i}=\tilde{k}_{1} \tilde{x}_{i}, \chi_{p-1}=\tilde{k}_{2} \tilde{y}\right)$ we find $\lambda_{1}=\lambda_{2}:=\lambda$ and they backreact on metric and $\varphi$ at quadratic order as

$$
\sim r^{\beta}, \quad \text { where } \beta:=2+\varphi_{0} \lambda,
$$

which gives a constraint on $\varphi_{0} \lambda$ because $\beta$ should be positive(negative) if the IR is at $r \rightarrow 0(\infty)$. This constraint (inequality) was summarized in the third column in (A.10) below. After considering all conditions (2.17)-(2.20), we find that the parameter space of $z, \theta$ are

$$
\begin{array}{rlr}
z<0, & \theta>p-1, & \varphi_{0} \lambda>-2, \\
1<z \leq 2, & \theta<(z-1)(p-1), & \varphi_{0} \lambda<-2, \\
2<z, & \theta<p-1, & \varphi_{0} \lambda<-2,
\end{array}
$$

which is the same as the case I for $\xi=1$ and represented in figure $1(\mathrm{~d}) . \lambda$ should be chosen for given $z, \theta$ to satisfy the last inequality in (A.10) with (A.8). If $\lambda$ is given, $\varphi_{0}$ should be chosen to satisfy the last inequality in (A.10), which further restricts the range of $z$ and $\theta$.

All formulas in this section are consistent with the formulas in Class I with replacements: $\xi=1, \lambda_{1}=\lambda_{2}$ and $\tilde{k}_{1}=\tilde{k}_{2}=0$.

\section{A.2 Class IV: irrelevant charge}

This class correspond to $\tilde{k}_{1}=\tilde{k}_{2}=0=a_{0}$ in the leading order so we start with an ansatz

$$
\begin{aligned}
\mathrm{d} s^{2} & =r^{\frac{2 \theta}{p-1}}\left[-\frac{\mathrm{d} t^{2}}{r^{2 z}}+\frac{L_{r}^{2} \mathrm{~d} r^{2}}{r^{2}}+\frac{\sum_{i=1}^{p-2} \mathrm{~d} \tilde{x}_{i}^{2}}{r^{2}}+\frac{\mathrm{d} \tilde{y}^{2}}{r^{2 \xi}}\right] \\
\varphi & =\varphi_{0} \log r, \quad A=0, \quad \chi_{i}=0, \quad \chi_{p-1}=0
\end{aligned}
$$

where the solution variables are determined by the action variable $\left(\delta, V_{0}\right)$ as follows:

$$
\begin{array}{rlrl}
z=1, & \xi=1, & \varphi_{0} & =\frac{2 \delta(p-1)}{\delta^{2}(p-1)-2}, \\
\theta=\frac{(p-1) \delta \varphi_{0}}{2}=\frac{\delta^{2}(p-1)^{2}}{\delta^{2}(p-1)-2}, & L_{r}^{2} & =\frac{(p-\theta)(p-1-\theta)}{V_{0}} .
\end{array}
$$

We may deduce $z=1$ from (A.6) by setting $a_{0}=0$. The relation between $\varphi_{0}$ and $\delta$ may be understood by requiring $z=1$ in the first equation of (A.2). $L_{r}$ is can be read from (A.5) with $z=1$. The action variable $\delta$ reads

$$
\delta=\frac{2 \theta}{(p-1) \varphi_{0}} \quad \text { with } \quad \varphi_{0}^{2}=\frac{2 \theta(1-p+\theta)}{p-1} .
$$

in terms of solution variables. 
Note that all formulas so far are independent of $\lambda_{1}, \lambda_{2}$ and $\gamma$ because both axions and charge are irrelevant. By turning on the subleading gauge field mode

$$
A_{t}(r)=a_{0} r^{\zeta-1}
$$

we find

$$
\zeta=p-1-\frac{p-3}{p-1} \theta-\gamma \varphi_{0}
$$

where $\zeta$ is a function of a free action parameter $\gamma$. This gauge field mode backreact on metric and $\varphi$ at quadratic order as

$$
\sim r^{\beta}, \quad \text { where } \beta:=p-1+\zeta-\theta .
$$

This mode analysis is parallel to class II from (2.45) to (2.47). If we turn on the subleading axion mode $\left(\chi_{i}=\tilde{k}_{1} \tilde{x}_{i}, \chi_{p-1}=\tilde{k}_{2} \tilde{y}\right)$ we find $\lambda_{1}=\lambda_{2}:=\lambda$ and they backreact on metric and $\varphi$ at quadratic order as

$$
\sim r^{\beta}, \quad \text { where } \beta:=2+\varphi_{0} \lambda .
$$

This mode analysis is parallel to (A.9) in class III.

After considering all conditions we listed in case I with (A.17) and (A.18), we find that the parameter space is

$$
z=1, \quad \theta<0, \quad \zeta<\theta+1-p, \quad \varphi_{0} \lambda<-2 .
$$

\section{B Marginally relevant and irrelevant axion}

In this subsection we consider the case that only one of the $k_{i}$ is nonzero. This is a hybrid of the class I and III $\left(a_{0} \neq 0\right)$; and the class II and IV $\left(a_{0}=0\right)$. The class I-i and II-i means $k_{1}$ is nonzero and the class I-ii and II-ii means $k_{2}$ is nonzero.

\section{B.1 Class I-i: marginally relevant charge}

We assume that the classical solutions are written as

$$
\begin{aligned}
& \mathrm{d} s^{2}=r^{\frac{2 \theta}{p-1}}\left[-\frac{\mathrm{d} t^{2}}{r^{2 z}}+\frac{L_{r}^{2} \mathrm{~d} r^{2}}{r^{2}}+\frac{\sum_{i=1}^{p-2} \mathrm{~d} \tilde{x}_{i}^{2}}{r^{2}}+\frac{\mathrm{d} \tilde{y}^{2}}{r^{2 \xi}}\right], \\
& \varphi=\varphi_{0} \log r, \quad A=a_{0} r^{\zeta-z} \mathrm{~d} t, \quad \chi_{i}=\tilde{k}_{1} \tilde{x}_{i}, \quad \chi_{p-1}=0 .
\end{aligned}
$$

By the equations of motion, the 'exponent' solution-parameters $(z, \xi, \theta, \zeta)$ may be expressed in terms of action-parameters $\left(\delta, \lambda_{1}, \gamma\right)$ as

$$
\begin{aligned}
& z=-\frac{2+\gamma^{2}+((p-5) p+5) \delta^{2}+(p-2)\left(2\left(\delta+\lambda_{1}\right) \gamma+\lambda_{1}\left(2(p-2) \delta+(p-1) \lambda_{1}\right)\right)}{(\gamma-\delta) \lambda_{1}}, \\
& \theta=\frac{(1-p) \delta}{\lambda_{1}}, \quad \xi=-\frac{\gamma+(p-2)\left(\delta+\lambda_{1}\right)}{\lambda_{1}}, \quad \zeta=\frac{\gamma-\delta}{\lambda_{1}} .
\end{aligned}
$$


Note that $\lambda_{2}$ does not contribute to the IR solution because $\tilde{k}_{2}=0$. A quick way to see this solutions is to solve (2.30) for $\lambda_{2}$ and plugging it to (2.28) making it $\lambda_{2}$ independent. They are not all independent and there is a constraint between solution-parameters $(\theta, \zeta, \xi)$

$$
\zeta=-(p-2-\theta)-\xi \equiv-\left(d_{\theta}-1\right)-\xi \equiv \zeta_{I},
$$

which does not give any relation between action-parameters contrary to (2.30). The 'coefficient' parameters are solved as

$$
\begin{aligned}
\varphi_{0} & =-\frac{2}{\lambda_{1}}, \\
L_{r}^{2} & =\frac{(p-2+z-\theta+\xi)(z-\theta+(p-2) \xi)}{V_{0}}, \\
a_{0}^{2} & =\frac{2(z-\xi)}{p-2+z-\theta+\xi}, \\
\tilde{k}_{1}^{2} & =\frac{2 V_{0}(\xi-1)}{z-\theta+(p-2) \xi} .
\end{aligned}
$$

It can be understood by $(2.35)-(2.37)$ by solving for $\tilde{k}_{1}$ with $\tilde{k}_{2}=0$ and plugging it back.

The action-parameters $\left(\delta, \lambda_{1}, \gamma\right)$ may be written in terms of solution-parameters $(z, \theta, \xi)$ :

$$
\delta=\frac{2 \theta}{(p-1) \varphi_{0}}, \quad \lambda_{1}=\frac{-2}{\varphi_{0}}, \quad \gamma=\frac{-2 \zeta+\frac{2}{p-1} \theta}{\varphi_{0}}
$$

where

$$
\begin{aligned}
\varphi_{0}^{2} & =2\left(\frac{\theta^{2}}{p-1}-z \zeta+2-p-\xi^{2}\right) \\
& =\frac{2(p-1-\theta)(1+p(z-1)-z-\theta)}{p-1}-2(\xi-1)(\xi-z+1),
\end{aligned}
$$

where $\zeta$ can be replaced by (B.2).

Here, all formulas are independent of $\lambda_{2}$. By considering the sub-leading axion mode $\tilde{k}_{2} \tilde{y}$ we find that it backreacts on metric and $\varphi$ at quadratic order as

$$
\sim r^{\beta}, \quad \text { where } \beta:=\varphi_{0} \lambda_{2}+2 \xi,
$$

which gives a constraint on $\varphi_{0} \lambda_{2}$ because $\beta$ should be positive(negative) if the IR is at $r \rightarrow 0(\infty)$. This and all other conditions (2.17)-(2.20) give us the parameter space shown in table 7 .

\section{B.2 Class I-ii: marginally relevant charge}

We assume that the classical solutions are written as

$$
\begin{aligned}
\mathrm{d} s^{2} & =r^{\frac{2 \theta}{p-1}}\left[-\frac{\mathrm{d} t^{2}}{r^{2 z}}+\frac{L_{r}^{2} \mathrm{~d} r^{2}}{r^{2}}+\frac{\sum_{i=1}^{p-2} \mathrm{~d} \tilde{x}_{i}^{2}}{r^{2}}+\frac{\mathrm{d} \tilde{y}^{2}}{r^{2 \xi}}\right] \\
\varphi & =\varphi_{0} \log r, \quad A=a_{0} r^{\zeta-z} \mathrm{~d} t, \quad \chi_{i}=0, \quad \chi_{p-1}=\tilde{k}_{2} \tilde{y} .
\end{aligned}
$$




\begin{tabular}{|c|c|c|}
\hline \multicolumn{3}{|c|}{$\xi \leq 0$} \\
\hline$z<\xi$ & $\theta>p-1$ & $\zeta=2-p+\theta-\xi, \quad \frac{\lambda_{2}}{\lambda_{1}}<\xi$ \\
\hline \multicolumn{3}{|c|}{$0<\xi<1$} \\
\hline$z<0$ & $\theta>p-1$ & $\zeta=2-p+\theta-\xi, \quad \frac{\lambda_{2}}{\lambda_{1}}<\xi$ \\
\hline \multicolumn{3}{|c|}{$1 \leq \xi$} \\
\hline$\xi<z \leq 1+\xi$ & $\theta<\frac{p-1}{2}\left(z-\sqrt{(z-2)^{2}+\frac{4(\xi-1)(-z+1+\xi)}{p-1}}\right)$ & $\zeta=2-p+\theta-\xi, \quad \frac{\lambda_{2}}{\lambda_{1}}>\xi$ \\
\hline $1+\xi<z$ & $\theta<p-1$ & $\zeta=2-p+\theta-\xi, \quad \frac{\lambda_{2}}{\lambda_{1}}>\xi$ \\
\hline
\end{tabular}

Table 7. Parameter range: Class I-i, $V_{0}>0$.

By the equations of motion, the 'exponent' solution-parameters $(z, \xi, \theta, \zeta)$ may be expressed in terms of action-parameters $\left(\delta, \lambda_{2}, \gamma\right)$ as

$$
\begin{aligned}
& z=\frac{\gamma^{2}+2(p-2)+2 \gamma\left(\lambda_{2}+(p-2) \delta\right)-(p-2) \delta^{2}+\lambda_{2}\left(2(p-2) \delta+(p-1) \lambda_{2}\right)}{(\gamma-\delta)\left(\gamma+\lambda_{2}+(p-2) \delta\right)}, \\
& \theta=\frac{(p-1)(p-2) \delta}{\gamma+\lambda_{2}+(p-2) \delta}, \quad \xi=-\frac{(p-2) \lambda_{2}}{\gamma+\lambda_{2}+(p-2) \delta}, \quad \zeta=-\frac{(p-2)(\gamma-\delta)}{\gamma+\lambda_{2}+(p-2) \delta}
\end{aligned}
$$

Note that $\lambda_{1}$ does not contribute to the IR solution because $\tilde{k}_{1}=0$. A quick way to see this solutions is to solve (2.30) for $\lambda_{1}$ and plugging it to (2.28) making it $\lambda_{1}$ independent. They are not all independent and there is a constraint between solution-parameters $(\theta, \zeta, \xi)$

$$
\zeta=-(p-2-\theta)-\xi \equiv-\left(d_{\theta}-1\right)-\xi \equiv \zeta_{I} .
$$

which does not give any relation between action-parameters contrary to (2.30). The 'coefficient' parameters are solved as

$$
\begin{aligned}
\varphi_{0} & =-\frac{2 \xi}{\lambda_{2}}, \\
L_{r}^{2} & =\frac{(p-2+z-\theta)(p-2+z-\theta+\xi)}{V_{0}}, \\
a_{0}^{2} & =\frac{2(z-1)}{p-2+z-\theta+\xi}, \\
\tilde{k}_{2}^{2} & =\frac{2 V_{0}(1-\xi)}{p-2+z-\theta} .
\end{aligned}
$$

It can be understood by $(2.35)-(2.37)$ by solving for $\tilde{k}_{2}$ with $\tilde{k}_{1}=0$ and plugging it back.

The action-parameters $\left(\delta, \lambda_{2}, \gamma\right)$ may be written in terms of solution-parameters $(z, \theta, \xi)$ :

$$
\delta=\frac{2 \theta}{(p-1) \varphi_{0}}, \quad \lambda_{2}=\frac{-2 \xi}{\varphi_{0}}, \quad \gamma=\frac{-2 \zeta+\frac{2}{p-1} \theta}{\varphi_{0}},
$$

where

$$
\begin{aligned}
\varphi_{0}^{2} & =2\left(\frac{\theta^{2}}{p-1}-z \zeta+2-p-\xi^{2}\right) \\
& =\frac{2(p-1-\theta)(1+p(z-1)-z-\theta)}{p-1}-2(\xi-1)(\xi-z+1),
\end{aligned}
$$

where $\zeta$ can be replaced by (B.12). 


\begin{tabular}{|c|c|c|}
\hline \multicolumn{3}{|c|}{$\xi \leq 0$} \\
\hline $1<z$ & $\theta<\xi(p-1)$ & $\zeta=2-p+\theta-\xi, \quad \frac{\lambda_{1}}{\lambda_{2}}<\xi^{-1}$ \\
\hline \multicolumn{3}{|c|}{$0<\xi<1$} \\
\hline $1<z \leq 1+\xi$ & $\theta<\frac{p-1}{2}\left(z-\sqrt{(z-2)^{2}+\frac{4(\xi-1)(-z+1+\xi)}{p-1}}\right)$ & $\zeta=2-p+\theta-\xi, \quad \frac{\lambda_{1}}{\lambda_{2}}>\xi^{-1}$ \\
\hline $1+\xi<z$ & $\theta<\xi(p-1)$ & $\zeta=2-p+\theta-\xi, \quad \frac{\lambda_{1}}{\lambda_{2}}>\xi^{-1}$ \\
\hline \multicolumn{3}{|c|}{$1 \leq \xi$} \\
\hline$z<0$ & $\theta>\xi(p-1)$ & $\zeta=2-p+\theta-\xi, \quad \frac{\lambda_{1}}{\lambda_{2}}<\xi^{-1}$ \\
\hline
\end{tabular}

Table 8. Parameter range: Class I-ii, $V_{0}>0$.

Similarly to the case I-i, all formulas here are independent of $\lambda_{1}$. By considering the sub-leading axion mode $\tilde{k}_{1} \tilde{x}_{i}$ we find that it backreacts on metric and $\varphi$ at quadratic order as

$$
\sim r^{\beta}, \quad \text { where } \beta:=\varphi_{0} \lambda_{1}+2,
$$

which gives a constraint on $\varphi_{0} \lambda_{1}$ because $\beta$ should be positive(negative) if the IR is at $r \rightarrow 0(\infty)$. This and all other conditions (2.17)-(2.20) give us the parameter space shown in table 8 .

\section{B.3 Class II-i: irrelevant charge}

We assume that the classical solutions are written as

$$
\begin{aligned}
\mathrm{d} s^{2} & =r^{\frac{2 \theta}{p-1}}\left[-\frac{\mathrm{d} t^{2}}{r^{2 z}}+\frac{L_{r}^{2} \mathrm{~d} r^{2}}{r^{2}}+\frac{\sum_{i=1}^{p-2} \mathrm{~d} \tilde{x}_{i}^{2}}{r^{2}}+\frac{\mathrm{d} \tilde{y}^{2}}{r^{2 \xi}}\right], \\
\varphi & =\varphi_{0} \log r, \quad A=0, \quad \chi_{i}=\tilde{k}_{1} \tilde{x}_{i}, \quad \chi_{p-1}=0 .
\end{aligned}
$$

By the equations of motion, the 'exponent' solution-parameters $(z, \xi, \theta)$ may be expressed in terms of action-parameters $\left(\delta, \lambda_{1}\right)$ as

$$
z=\xi, \quad \theta=\frac{(1-p) \delta}{\lambda_{1}}, \quad \xi=\frac{2-(p-1) \delta^{2}+(p-2) \lambda_{1}^{2}}{\left((p-1) \delta+(p-2) \lambda_{1}\right) \lambda_{1}} .
$$

Note that $\lambda_{2}$ and $\gamma$ does not contribute to the IR solution because $\tilde{k}_{2}=a_{0}=0$. The first equation can be understood by $(2.43)$ where we set $\tilde{k}_{2}=0$. The 'coefficient' parameters are solved as

$$
\begin{aligned}
\varphi_{0} & =-\frac{2}{\lambda_{1}}, \\
L_{r}^{2} & =\frac{(p-2-\theta+2 \xi)((p-1) \xi-\theta)}{V_{0}}, \\
\tilde{k}_{1}^{2} & =\frac{2 V_{0}(\xi-1)}{(p-1) \xi-\theta} .
\end{aligned}
$$

It can be understood by (2.43) by plugging $z=\xi$. 


\begin{tabular}{|cccc|}
\hline \multicolumn{4}{|c|}{$\xi<0$} \\
\hline$z=\xi$ & $\theta>p-1$ & $\zeta>2-p+\theta-\xi$, & $\frac{\lambda_{2}}{\lambda_{1}}<\xi$ \\
\hline \multicolumn{4}{|c|}{$1<\xi$} \\
\hline$z=\xi$ & $\theta<\frac{p-1}{2}\left(\xi-\sqrt{(\xi-2)^{2}+\frac{4(\xi-1)}{p-1}}\right)$ & $\zeta<2-p+\theta-\xi$, & $\frac{\lambda_{2}}{\lambda_{1}}>\xi$ \\
\hline
\end{tabular}

Table 9. Parameter range: Class II-i, $V_{0}>0$.

The action-parameters $\left(\delta, \lambda_{1}\right)$ may be written in terms of solution-parameters $(\theta, \xi)$ :

$$
\delta=\frac{2 \theta}{(p-1) \varphi_{0}}, \quad \lambda_{1}=\frac{-2}{\varphi_{0}},
$$

where

$$
\varphi_{0}^{2}=\frac{2(p-1-\theta)(1+p(\xi-1)-\xi-\theta)}{p-1}-2(\xi-1) .
$$

Note that all formulas so far are independent of $\lambda_{2}$ and $\gamma$ because one axion $\left(\tilde{k}_{2} \tilde{y}\right)$ and the charge are irrelevant. By turning on the subleading gauge field mode

$$
A_{t}(r)=a_{0} r^{\zeta-\xi}
$$

we find

$$
\zeta=\frac{(p(p-3)+2(\theta+1))+(1-p)\left(\gamma \varphi_{0}-\xi+\theta\right)}{p-1},
$$

where $\zeta$ is a function of a free action parameter $\gamma$. This gauge field mode backreact on metric and $\varphi$ at quadratic order as

$$
\sim r^{\beta_{1}}, \quad \text { where } \quad \beta_{1}:=p-2+\zeta-\theta+\xi .
$$

If we turn on the subleading axion mode $\left(\chi_{p-1}=\tilde{k}_{2} \tilde{y}\right)$ we find

$$
\lambda_{2}=\frac{p-2+\zeta-\theta-\xi}{\varphi_{0}},
$$

and they backreact on metric and $\varphi$ at quadratic order as

$$
\sim r^{\beta_{2}}, \quad \text { where } \beta_{2}:=\varphi_{0} \lambda_{2}+2 \xi .
$$

After considering all conditions (2.17)-(2.20) with the conditions for (B.29) and (B.31), we find that the parameter space which is shown in table 9 .

\section{B.4 Class II-ii: irrelevant charge}

We assume that the classical solutions are written as

$$
\begin{aligned}
\mathrm{d} s^{2} & =r^{\frac{2 \theta}{p-1}}\left[-\frac{\mathrm{d} t^{2}}{r^{2 z}}+\frac{L_{r}^{2} \mathrm{~d} r^{2}}{r^{2}}+\frac{\sum_{i=1}^{p-2} \mathrm{~d} \tilde{x}_{i}^{2}}{r^{2}}+\frac{\mathrm{d} \tilde{y}^{2}}{r^{2 \xi}}\right], \\
\varphi & =\varphi_{0} \log r, \quad A=0, \quad \chi_{i}=0, \quad \chi_{p-1}=\tilde{k}_{2} \tilde{y} .
\end{aligned}
$$


By the equations of motion, the 'exponent' solution-parameters $(z, \xi, \theta)$ may be expressed in terms of action-parameters $\left(\delta, \lambda_{1}\right)$ as

$$
z=1, \quad \theta=\frac{(1-p)\left(\lambda_{2}+(p-1) \delta\right) \delta}{2+\lambda_{2}^{2}-(p-1) \delta^{2}}, \quad \xi=\frac{\lambda_{2}\left(\lambda_{2}+(p-1) \delta\right)}{2+\lambda_{2}^{2}-(p-1) \delta^{2}} .
$$

Note that $\lambda_{1}$ and $\gamma$ does not contribute to the IR solution because $\tilde{k}_{1}=a_{0}=0$. The first equation can be understood by $(2.43)$ where we set $\tilde{k}_{1}=0$. The 'coefficient' parameters are solved as

$$
\begin{aligned}
\varphi_{0} & =-\frac{2 \xi}{\lambda_{2}}, \\
L_{r}^{2} & =\frac{(p-1-\theta)(p-1-\theta+\xi)}{V_{0}}, \\
\tilde{k}_{2}^{2} & =\frac{2 V_{0}(\xi-1)}{\theta-(p-1)} .
\end{aligned}
$$

It can be understood by (2.43) by plugging $z=1$.

The action-parameters $\left(\delta, \lambda_{2}\right)$ may be written in terms of solution-parameters $(\theta, \xi)$ :

$$
\delta=\frac{2 \theta}{(p-1) \varphi_{0}}, \quad \lambda_{2}=-\frac{2 \xi}{\varphi_{0}}
$$

where

$$
\varphi_{0}^{2}=2 \theta\left(\frac{\theta}{p-1}-1\right)-2(\xi-1) \xi
$$

Note that all formulas so far are independent of $\lambda_{1}$ and $\gamma$ because one axion $\left(k_{1} x_{i}\right)$ and the charge are irrelevant. By turning on the subleading gauge field mode

$$
A_{t}(r)=a_{0} r^{\zeta-1}
$$

we find

$$
\zeta=\frac{2+\gamma \varphi_{0}+3 \theta-\xi+p\left(p-3-\gamma \varphi_{0}-\theta+\xi\right)}{p-1}
$$

where $\zeta$ is a function of a free action parameter $\gamma$. This gauge field mode backreact on metric and $\varphi$ at quadratic order as

$$
\sim r^{\beta_{1}}, \quad \text { where } \quad \beta_{1}:=p-2+\zeta-\theta+\xi .
$$

If we turn on the subleading axion mode $\left(\tilde{k}_{1} \tilde{x}_{i}\right)$ we find

$$
\lambda_{1}=\frac{p-4+\zeta-\theta+\xi}{\varphi_{0}},
$$

and they backreact on metric and $\varphi$ at quadratic order as

$$
\sim r^{\beta_{2}}, \quad \text { where } \beta_{2}:=\varphi_{0} \lambda_{1}+2 .
$$

After considering all conditions (2.17)-(2.20) with the conditions for (B.41) and (B.43), we find that the parameter space which is shown in table 10 . 


\begin{tabular}{|cccc|}
\hline \multicolumn{4}{|c|}{$\xi \leq 0$} \\
\hline$z=1$ & $\theta<\xi(p-1)$ & $\zeta<2-p+\theta-\xi$, & $\frac{\lambda_{1}}{\lambda_{2}}<\xi^{-1}$ \\
\hline \multicolumn{4}{|c|}{$0<\xi<1$} \\
\hline$z=1$ & $\theta<\frac{p-1}{2}\left(1-\sqrt{1+\frac{4(\xi-1) \xi}{p-1}}\right)$ & $\zeta<2-p+\theta-\xi$, & $\frac{\lambda_{1}}{\lambda_{2}}>\xi^{-1}$ \\
\hline
\end{tabular}

Table 10. Parameter range: Class II-ii, $V_{0}>0$.

\section{Consistency check by coordinate transformation}

In order to compare the results in section 3 with [34], let us summarize the main formulas in [34]. The metric is written as

$$
\mathrm{d} s^{2}=-U(\bar{r}) \mathrm{d} \vec{t}^{2}+\frac{\mathrm{d} \bar{r}^{2}}{U(\bar{r})}+h_{1}(\bar{r}) \mathrm{d} \bar{x}^{2}+h_{2}(\bar{r}) \mathrm{d} \bar{y}^{2},
$$

of which IR geometry is parameterized as

$$
U(\bar{r})=L_{t}^{-2} \bar{r}^{u_{1}}\left(1-\frac{\bar{r}_{h}^{\Delta}}{\bar{r}^{\Delta}}\right), \quad h_{1}(\bar{r})=L_{x}^{-2} \bar{r}^{2 v_{1}}, \quad h_{2}(\bar{r})=L_{y}^{-2} \bar{r}^{2 v_{2}},
$$

where $\Delta=v_{1}+v_{2}+u_{1}-1$. The parameters $\mathbb{E}_{x}$ and $\mathbb{E}_{y}$ are obtained as

$$
\mathbb{E}_{x}=\frac{D_{T, x}}{v_{B, x}^{2} \tau_{L}}=\frac{u_{1}-1}{u_{1}-2 v_{1}}, \quad \mathbb{E}_{y}=\frac{D_{T, y}}{v_{B, y}^{2} \tau_{L}}=\frac{u_{1}-1}{u_{1}-2 v_{2}} .
$$

As a consistency check, we may consider the coordinate transformation between (C.1) and our metric (3.19)

$$
\mathrm{d} s^{2}=r^{\theta}\left(-f(r) \frac{\mathrm{d} t^{2}}{r^{2 z}}+\frac{L_{r}^{2} \mathrm{~d} r^{2}}{f(r) r^{2}}+\frac{L_{1}^{2} \mathrm{~d} x^{2}}{r^{2}}+\frac{L_{2}^{2} \mathrm{~d} y^{2}}{r^{2 \xi}}\right) .
$$

Two metrics are related as follows.

$$
\begin{aligned}
& \{\bar{t}, \bar{r}, \bar{x}, \bar{y}\}=\left\{t, \frac{L_{r}}{\theta-z} r^{\theta-z}, x, y\right\} \\
& u_{1}=\frac{\theta-2 z}{\theta-z}, \quad 2 v_{1}=\frac{\theta-2}{\theta-z}, \quad 2 v_{2}=\frac{\theta-2 \xi}{\theta-z}, \\
& L_{t}^{2}=\left(\frac{L_{r}}{\theta-z}\right)^{\frac{2 z-\theta}{z-\theta}}, \quad L_{x}^{2}=\frac{1}{L_{1}^{2}}\left(\frac{L_{r}}{\theta-z}\right)^{\frac{\theta-2}{\theta-z}}, \quad L_{y}^{2}=\frac{1}{L_{2}^{2}}\left(\frac{L_{r}}{\theta-z}\right)^{\frac{\theta-2 \xi}{\theta-z}} .
\end{aligned}
$$

We have confirmed all of our results agree to [34] by using this coordinate transformation. For example,

$$
\mathbb{E}_{x}=\frac{z}{2 z-2}=\frac{u_{1}-1}{u_{1}-2 v_{1}}, \quad \mathbb{E}_{y}=\frac{z}{2 z-2 \xi}=\frac{u_{1}-1}{u_{1}-2 v_{2}} .
$$

Open Access. This article is distributed under the terms of the Creative Commons Attribution License (CC-BY 4.0), which permits any use, distribution and reproduction in any medium, provided the original author(s) and source are credited. 


\section{References}

[1] S.A. Hartnoll, A. Lucas and S. Sachdev, Holographic quantum matter, arXiv:1612.07324 [INSPIRE].

[2] C.C. Homes et al., Universal scaling relation in high-temperature superconductors, Nature 430 (2004) 539 [cond-mat/0404216] [INSPIRE].

[3] J. Zaanen, Superconductivity: Why the temperature is high, Nature 430 (2004) 512.

[4] J. Erdmenger, B. Herwerth, S. Klug, R. Meyer and K. Schalm, S-Wave Superconductivity in Anisotropic Holographic Insulators, JHEP 05 (2015) 094 [arXiv:1501.07615] [INSPIRE].

[5] J. Zaanen, Y.-W. Sun, Y. Liu and K. Schalm, Holographic Duality in Condensed Matter Physics, Cambridge University Press (2015).

[6] M. Ammon and J. Erdmenger, Gauge/gravity duality, Cambridge University Press, Cambridge, U.K. (2015)

[7] K.-Y. Kim, K.K. Kim and M. Park, A Simple Holographic Superconductor with Momentum Relaxation, JHEP 04 (2015) 152 [arXiv: 1501.00446] [INSPIRE].

[8] K.K. Kim, M. Park and K.-Y. Kim, Ward identity and Homes' law in a holographic superconductor with momentum relaxation, JHEP 10 (2016) 041 [arXiv:1604.06205] [INSPIRE].

[9] K.-Y. Kim and C. Niu, Homes' law in Holographic Superconductor with Q-lattices, JHEP 10 (2016) 144 [arXiv: 1608.04653] [INSPIRE].

[10] S.A. Hartnoll, Theory of universal incoherent metallic transport, Nature Phys. 11 (2015) 54 [arXiv: 1405.3651] [INSPIRE].

[11] M. Blake, Universal Charge Diffusion and the Butterfly Effect in Holographic Theories, Phys. Rev. Lett. 117 (2016) 091601 [arXiv:1603.08510] [INSPIRE].

[12] M. Blake, Universal Diffusion in Incoherent Black Holes, Phys. Rev. D 94 (2016) 086014 [arXiv: 1604.01754] [INSPIRE].

[13] A. Lucas and J. Steinberg, Charge diffusion and the butterfly effect in striped holographic matter, JHEP 10 (2016) 143 [arXiv:1608.03286] [INSPIRE].

[14] R.A. Davison, W. Fu, A. Georges, Y. Gu, K. Jensen and S. Sachdev, Thermoelectric transport in disordered metals without quasiparticles: The Sachdev-Ye-Kitaev models and holography, Phys. Rev. B 95 (2017) 155131 [arXiv:1612.00849] [INSPIRE].

[15] M. Baggioli, B. Goutéraux, E. Kiritsis and W.-J. Li, Higher derivative corrections to incoherent metallic transport in holography, JHEP 03 (2017) 170 [arXiv:1612.05500] [INSPIRE].

[16] K.-Y. Kim and C. Niu, Diffusion and Butterfly Velocity at Finite Density, JHEP 06 (2017) 030 [arXiv: 1704.00947] [INSPIRE].

[17] Y. Sekino and L. Susskind, Fast Scramblers, JHEP 10 (2008) 065 [arXiv:0808.2096] [INSPIRE].

[18] S.H. Shenker and D. Stanford, Black holes and the butterfly effect, JHEP 03 (2014) 067 [arXiv:1306.0622] [INSPIRE].

[19] D.A. Roberts, D. Stanford and L. Susskind, Localized shocks, JHEP 03 (2015) 051 [arXiv:1409.8180] [INSPIRE]. 
[20] J. Maldacena, S.H. Shenker and D. Stanford, A bound on chaos, JHEP 08 (2016) 106 [arXiv: 1503.01409] [INSPIRE].

[21] D.A. Roberts and B. Swingle, Lieb-Robinson Bound and the Butterfly Effect in Quantum Field Theories, Phys. Rev. Lett. 117 (2016) 091602 [arXiv:1603.09298] [INSPIRE].

[22] Y. Ling, P. Liu and J.-P. Wu, Holographic Butterfly Effect at Quantum Critical Points, JHEP 10 (2017) 025 [arXiv:1610.02669] [INSPIRE].

[23] M. Alishahiha, A. Davody, A. Naseh and S.F. Taghavi, On Butterfly effect in Higher Derivative Gravities, JHEP 11 (2016) 032 [arXiv:1610.02890] [InSPIRE].

[24] M.M. Qaemmaqami, Butterfly effect in 3D gravity, Phys. Rev. D 96 (2017) 106012 [arXiv: 1707.00509] [INSPIRE].

[25] V. Jahnke, Delocalizing Entanglement of Anisotropic Black Branes, arXiv:1708.07243 [INSPIRE].

[26] S. Sachdev and B. Keimer, Quantum Criticality, Phys. Today 64N2 (2011) 29 [arXiv:1102.4628] [INSPIRE].

[27] J.A.N. Bruin, H. Sakai, R.S. Perry and A.P. Mackenzie, Similarity of scattering rates in metals showing t-linear resistivity, Science 339 (2013) 804.

[28] J.C. Zhang et al., Anomalous Thermal Diffusivity in Underdoped $\mathrm{YBa}_{2} \mathrm{Cu}_{3} \mathrm{O}_{6+x}$, Proc. Nat. Acad. Sci. 114 (2017) 5378 [arXiv: 1610.05845] [INSPIRE].

[29] I.L. Aleiner, L. Faoro and L.B. Ioffe, Microscopic model of quantum butterfly effect: out-of-time-order correlators and traveling combustion waves, Annals Phys. 375 (2016) 378 [arXiv: 1609.01251] [INSPIRE].

[30] B. Swingle and D. Chowdhury, Slow scrambling in disordered quantum systems, Phys. Rev. B 95 (2017) 060201 [arXiv:1608.03280] [INSPIRE].

[31] A.A. Patel and S. Sachdev, Quantum chaos on a critical Fermi surface, Proc. Nat. Acad. Sci. 114 (2017) 1844 [arXiv:1611.00003] [INSPIRE].

[32] B. Goutéraux, Charge transport in holography with momentum dissipation, JHEP 04 (2014) 181 [arXiv: 1401.5436] [INSPIRE].

[33] M. Blake and A. Donos, Diffusion and Chaos from near AdS 2 horizons, JHEP 02 (2017) 013 [arXiv:1611.09380] [INSPIRE].

[34] M. Blake, R.A. Davison and S. Sachdev, Thermal diffusivity and chaos in metals without quasiparticles, Phys. Rev. D 96 (2017) 106008 [arXiv:1705.07896] [INSPIRE].

[35] S.-F. Wu, B. Wang, X.-H. Ge and Y. Tian, Collective diffusion and strange-metal transport, arXiv: 1702.08803 [INSPIRE].

[36] M. Baggioli and W.-J. Li, Diffusivities bounds and chaos in holographic Horndeski theories, JHEP 07 (2017) 055 [arXiv: 1705.01766] [INSPIRE].

[37] A. Bohrdt, C.B. Mendl, M. Endres and M. Knap, Scrambling and thermalization in a diffusive quantum many-body system, New J. Phys. 19 (2017) 063001 [arXiv:1612.02434] [INSPIRE].

[38] Y. Werman, S.A. Kivelson and E. Berg, Quantum chaos in an electron-phonon bad metal, arXiv: 1705.07895 [INSPIRE]. 
[39] Y. Gu, X.-L. Qi and D. Stanford, Local criticality, diffusion and chaos in generalized Sachdev-Ye-Kitaev models, JHEP 05 (2017) 125 [arXiv: 1609.07832] [INSPIRE].

[40] S.-K. Jian and H. Yao, Solvable Sachdev-Ye-Kitaev models in higher dimensions: from diffusion to many-body localization, Phys. Rev. Lett. 119 (2017) 206602 [arXiv:1703.02051] [INSPIRE].

[41] D. Giataganas, U. Gürsoy and J.F. Pedraza, Strongly-coupled anisotropic gauge theories and holography, arXiv: 1708.05691 [INSPIRE].

[42] Y. Gu, A. Lucas and X.-L. Qi, Energy diffusion and the butterfly effect in inhomogeneous Sachdev-Ye-Kitaev chains, SciPost Phys. 2 (2017) 018 [arXiv:1702.08462] [InSPIRE].

[43] T. Hartman, S.A. Hartnoll and R. Mahajan, Upper Bound on Diffusivity, Phys. Rev. Lett. 119 (2017) 141601 [arXiv:1706.00019] [INSPIRE].

[44] Y. Ling, Z. Xian and Z. Zhou, Power Law of Shear Viscosity in Einstein-Maxwell-Dilaton-Axion model, Chin. Phys. C 41 (2017) 023104 [arXiv: 1610.08823] [INSPIRE].

[45] Y.-Z. Li, H.-S. Liu and H. Lü, Quasi-Topological Ricci Polynomial Gravities, arXiv:1708.07198 [INSPIRE].

[46] P. Kovtun, D.T. Son and A.O. Starinets, Viscosity in strongly interacting quantum field theories from black hole physics, Phys. Rev. Lett. 94 (2005) 111601 [hep-th/0405231] [INSPIRE].

[47] M. Brigante, H. Liu, R.C. Myers, S. Shenker and S. Yaida, Viscosity Bound Violation in Higher Derivative Gravity, Phys. Rev. D 77 (2008) 126006 [arXiv:0712.0805] [INSPIRE].

[48] M. Brigante, H. Liu, R.C. Myers, S. Shenker and S. Yaida, The Viscosity Bound and Causality Violation, Phys. Rev. Lett. 100 (2008) 191601 [arXiv:0802.3318] [INSPIRE].

[49] S.A. Hartnoll, D.M. Ramirez and J.E. Santos, Entropy production, viscosity bounds and bumpy black holes, JHEP 03 (2016) 170 [arXiv:1601.02757] [INSPIRE].

[50] L. Alberte, M. Baggioli and O. Pujolàs, Viscosity bound violation in holographic solids and the viscoelastic response, JHEP 07 (2016) 074 [arXiv: 1601.03384] [INSPIRE].

[51] P. Burikham and N. Poovuttikul, Shear viscosity in holography and effective theory of transport without translational symmetry, Phys. Rev. D 94 (2016) 106001 [arXiv: 1601.04624] [INSPIRE].

[52] Y. Ling, Z.-Y. Xian and Z. Zhou, Holographic Shear Viscosity in Hyperscaling Violating Theories without Translational Invariance, JHEP 11 (2016) 007 [arXiv: 1605.03879] [INSPIRE].

[53] A. Rebhan and D. Steineder, Violation of the Holographic Viscosity Bound in a Strongly Coupled Anisotropic Plasma, Phys. Rev. Lett. 108 (2012) 021601 [arXiv:1110.6825] [INSPIRE].

[54] K.A. Mamo, Holographic RG flow of the shear viscosity to entropy density ratio in strongly coupled anisotropic plasma, JHEP 10 (2012) 070 [arXiv:1205.1797] [INSPIRE].

[55] S. Jain, R. Samanta and S.P. Trivedi, The Shear Viscosity in Anisotropic Phases, JHEP 10 (2015) 028 [arXiv: 1506.01899] [INSPIRE].

[56] S. Jain, N. Kundu, K. Sen, A. Sinha and S.P. Trivedi, A Strongly Coupled Anisotropic Fluid From Dilaton Driven Holography, JHEP 01 (2015) 005 [arXiv: 1406.4874] [INSPIRE]. 
[57] R.A. Davison and B. Goutéraux, Dissecting holographic conductivities, JHEP 09 (2015) 090 [arXiv: 1505.05092] [INSPIRE].

[58] E. Kiritsis and J. Ren, On Holographic Insulators and Supersolids, JHEP 09 (2015) 168 [arXiv: 1503.03481] [INSPIRE].

[59] A. Donos and J.P. Gauntlett, Thermoelectric DC conductivities from black hole horizons, JHEP 11 (2014) 081 [arXiv: 1406.4742] [INSPIRE].

[60] K.-Y. Kim, K.K. Kim, Y. Seo and S.-J. Sin, Coherent/incoherent metal transition in a holographic model, JHEP 12 (2014) 170 [arXiv:1409.8346] [INSPIRE].

[61] K.-Y. Kim, K.K. Kim, Y. Seo and S.-J. Sin, Gauge Invariance and Holographic Renormalization, Phys. Lett. B 749 (2015) 108 [arXiv:1502.02100] [INSPIRE].

[62] K.-Y. Kim, K.K. Kim, Y. Seo and S.-J. Sin, Thermoelectric Conductivities at Finite Magnetic Field and the Nernst Effect, JHEP 07 (2015) 027 [arXiv:1502.05386] [INSPIRE].

[63] M. Blake and D. Tong, Universal Resistivity from Holographic Massive Gravity, Phys. Rev. D 88 (2013) 106004 [arXiv:1308.4970] [InSPIRE].

[64] M. Blake, D. Tong and D. Vegh, Holographic Lattices Give the Graviton an Effective Mass, Phys. Rev. Lett. 112 (2014) 071602 [arXiv:1310.3832] [INSPIRE].

[65] A. Amoretti, M. Baggioli, N. Magnoli and D. Musso, Chasing the cuprates with dilatonic dyons, JHEP 06 (2016) 113 [arXiv: 1603.03029] [INSPIRE]. 